\title{
Next Generation Optical \\ Wireless Communication Systems \\ Using Fiber Direct Coupled Optical Antennas
}

\author{
Koichi Takahashi
}

Additional information is available at the end of the chapter

http://dx.doi.org/10.5772/48395

\section{Introduction}

The broadband communication environment becomes the essential social infrastructure when we perform a social activity. Above all, the optical fiber network was the main artery which supported a communications infrastructure, and various technology developments have been performed to take in traffic to increase rapidly [Cochennec 2002][ITU-T 2001][ ITU-T 2011]. On the other hand, wireless communication technology is essential element technology to realize ubiquitous communication environment. And new generation service development by new technology is beginning in the fields of mobile communication and broadcast [Lee 2007]. In addition, the research and development of ultra-high speed photonic network [Ben 2006][Zhang 2005][Kitayama 2005] performing the information transmission from the edge of the network to the edge in an optical domain is pushed forward, but an application of the radio technology to raise the flexibility in photonic network is an important problem, and advancement of optical wireless technology having possibility is expected [Kim 1999][Bouchet 1999]. However, it becomes further ultra-high speed/huge-capacity of the optical wireless and wavelength division multiplexing (WDM) limitation factor of the flexibility corresponding to various transmission methods because the current optical wireless system performs from optical to electrical $(\mathrm{O} / \mathrm{E})$ conversion of a transmitting and receiving part respectively. Therefore the realization of the optical wireless method that is not dependent on in ultra-high speed/huge-capacity and the interface (a protocol) of the device is enabled more than before if we can connect optical fiber to optical wireless as optical signal without performing $\mathrm{O} / \mathrm{E}$ conversion, and it is expected that it can be it with the effective means of flexible photonic network construction. It is anticipated that compact optical antennas with an advanced fine optical tracking system will be used for the ultra-fast free space optical communication system in the next generation. Such laser 
communication systems will be used not only for the space communications [Kaliski 1999][Borocom 2005][Koyama 2004] but also the terrestrial long-distance photonic network. In this paper, we discuss demand specifications of optical antennas considering phenomena such as the scintillation which occurs by atmospheric turbulence. And then we explain optics design and the design results to satisfy demand specifications. We also mention the fine tracking mechanism using the fine pointing mirror (FPM) and the feedback of the signal's incident angle detection by the quadrant detector (QD) for the antenna. The influences of fluctuation of laser beam angle-of-arrival (AOA) are reduced effectively. We mention the following research studies of the two optical wireless communication systems such as next generation free-space optical communication (NG-FSO) system and radio on FSO (RoFSO) system.

\section{Transition of the Free Space Optical Communication systems}

The various concepts and architectures of Free Space Optical Communication (FSO) systems are illustrated in Figure 1. Figure 1(a) shows a conventional FSO system [Infrared][ FreeSpace]. Since it uses as a transmission media the difference wavelength as an optical fiber, it includes optical and electrical conversion between an optical fiber side and the free-space side. Furthermore, it is necessary to prepare a different interface which performs individual modulation and demodulation, coding, etc. for every communications service. Figure 1(b) shows the concept of a NG-FSO system [Takahashi 2008][Matsumoto 2008][Kazaura 2007]. In seamless connection of free-space and fiber systems an optical beam is emitted directly from a single mode fiber (SMF) termination to free-space using a new concept FSO antenna. Loss of the optical signal power caused by space transmission can be compensated using a fiber amplifier using the same wavelength band of $1550 \mathrm{~nm}$ as an optical fiber network [Khaleghi 1996][ Luo 1998]. In this method the need to convert the optical signal from electrical to optical formats or vice versa for transmitting or receiving through space is eliminated. Figure 1(c) shows the concept of a Radio on Free Space Optical communication (RoFSO) system for realizing application in radio service combining with Radio on Fiber (RoF) technology [Al-Raweshidy 2002][ Hai 2006]. Main advantage and goal of RoFSO systems is they can be used to quickly and effectively provide heterogeneous wireless service [Komaki 2003][Tsukamoto 2006] for example WiFi (IEEE 802.11) ,WiMAX (IEEE 802.16), cellular based 3G signals etc, simultaneously.

However, the challenge in NG-FSO system design is making seamless connection of free space propagated beam to the SMF. The optical signal which has been propagated through free-space is reduced by the optical elements like lens etc. in the antenna and focused to the core of SMF. The optical beam transmitted through the free-space is influenced by various weather conditions, such as attenuation by rain, fog, snow etc., and by atmospheric absorption. Furthermore, the beam experiences atmospheric turbulence as it propagates through free-space, as well as vibrations of the device at the installation site and beam distortion occurrence. The consequence of these effects is the fluctuation of the beam AOA which in turn leads to significant variation in the power of the light focused into the SMF. 
Although AOA is not a problem for conventional FSO system thanks to a large area of the photo detector, it is a problem for NG-FSO because of little margin between the arrival beam and SMF. It is therefore difficult to maintain a stable link performance.

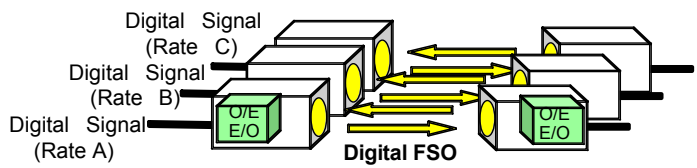

(a) Conventional FSO System

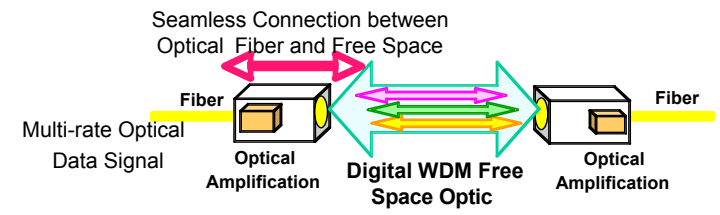

(b) Next Generation FSO System

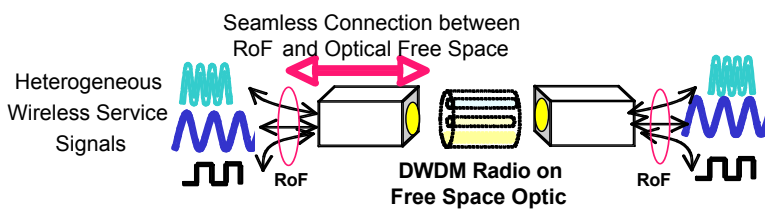

(c) Advanced DWDM RoFSO System

Figure 1. The concept of various FSO systems,

(a) conventional, (b) NG-FSO and (c) RoFSO

\section{Laser beam propagation in atmosphere}

When laser beam propagates in the atmosphere [Tatarskii 1971][Dayton 1992][ Hufnagel 1974][Friend 1967][Andrew 1998], its phase is fluctuated by the scatter of cloud, fog and rain, also by the change of local refractive index of the atmosphere. The fluctuation is varied depending on medium, and it becomes the largest when its size is similar to the size of light, also the medium constant changes spatially and temporally at random. When refractive index slowly and continuously changes due to atmospheric turbulence, the forward scattering dominates because the scale of fluctuation is larger than the wave length. In this case, it is possible to ignore the fluctuation of plane of polarization, but the fluctuation of phase/angle of arrival and the amplitude and the scintillation should be taken into consideration. On the other hand, when the medium is distributed discretely such as cloud, fog and rain and so on, the refractive index changes drastically. Therefore, the equivalent damping which occurs due to absorbing/scattering of particles and the influence against polarization property by multiple scattering with multiple particles should be considered. Modified von Karman spectrum density function is used not to overflow an integral value in theoretical analyses [Andrew 1995]: 


$$
\Phi_{n}(K)=0.033 C_{n}^{2}\left(K^{2}+\kappa_{0}^{2}\right)^{-\frac{11}{6}} \exp \left(-\frac{K^{2}}{\kappa_{i}^{2}}\right)
$$

Herein, $\kappa_{i}=5.92 / l_{0}, \quad \kappa_{0}=2 \pi / L_{0}$

$l_{0}$ is the inner scale, $L_{0}$ is the outer scale. Based on the actual measurements of the change of $C_{n}^{2}$ (refractive index structure constant) in high altitude, Hufnagel-Valley model $\left(\mathrm{H}-\mathrm{V}_{5 / 7}\right.$ model) parameterized by the scaling factors of wind speed in upper layer of the atmosphere and the size of the fluctuation of the atmosphere are used:

$$
C_{n}^{2}(h)=0.00594\left(\frac{v}{27}\right)^{2}\left(10^{-5} h\right)^{10} \exp \left(-\frac{h}{1000}\right)+2.7 \times 10^{-16} \exp \left(-\frac{h}{1500}\right)+A \exp \left(-\frac{h}{100}\right)
$$

Herein, $h$ represents the height of atmosphere $(\mathrm{m}), v$ represents the wind velocity in upper layer of atmosphere $(\mathrm{m} / \mathrm{s}), A$ is the scaling factor which represents the fluctuation of the earth's surface $\left(\mathrm{m}^{-2 / 3}\right)$. In $\mathrm{H}-\mathrm{V}$ day model, the scaling factors are $v=21 \mathrm{~m} / \mathrm{s}$ and $A=1.7 \times 10^{-14}$ $\mathrm{m}^{-2 / 3}$. This is equivalent to looking up the sky from the earth's surface, and the coherence length of the fluctuation of the atmosphere is $r_{0}=5 \mathrm{~cm}$. We firstly consider the averaged influence of fluctuation which also means the extensity of beam and degradation of the strength of the central part. We use $\mathrm{H}-\mathrm{V}$ model to represent the fluctuation of the refractive index of the atmosphere. And we calculate the averaged intensity of light of the receiving point when the Gaussian beam is transmitted from the earth to the sky (uplink). If the atmosphere is not disturbed and it is possible to apply Rytov approximation to the wave equation, the averaged strength of the Gaussian beam after propagating the distance $\mathrm{L}$ is :

$$
\langle I(r, L)\rangle=\frac{W_{0}^{2}}{W_{e}^{2}} \exp \left(-\frac{2 r^{2}}{W_{e}^{2}}\right)
$$

Therefore, the anticipated value of the received beam profile still remains Gaussian distribution, and the variant is given by the effective beam radius as follows:

$$
W_{e}=W\left(1+G_{u}\right)^{1 / 2}
$$

And,

$$
\begin{gathered}
G_{u}=4.35 \mu_{1} \Lambda^{5 / 6} k^{7 / 6}\left(H-h_{0}\right)^{5 / 6} \sec ^{11 / 6}(\xi) \\
\mu_{1}=\int_{h_{0}}^{H} C_{n}^{2}(h) \xi_{1}^{5 / 3} d h, \xi_{1}=1-\frac{h-h_{0}}{H-h_{0}}
\end{gathered}
$$

are the parameters which represents the influence of fluctuation, $W_{0}$ is the radius of transmitting beam, $\kappa$ is the wave number, $L$ is the distance between the transmitting system and the receiving system, $h_{0}$ is the height between the earth's surface and the 
transmitting system, $\xi$ is zenith angle and $H=h_{0}+L \cos (\xi)$ is the height of the receiving system in the sky. Also, $W$ is the radius without the fluctuation of the atmosphere, and it is possible to describe $W$ using $F_{0}$ (the curvature radius of exit point of the transmitting light) as follows:

$$
W=W_{0} \sqrt{\left(1-\frac{L}{F_{0}}\right)^{2}+\left(\frac{2 L}{k W_{0}^{2}}\right)^{2}}=W_{0} \sqrt{\Theta_{0}^{2}+\Lambda_{0}^{2}}
$$

Herein:

$$
\begin{gathered}
\Lambda=\frac{2 L}{k W^{2}}=\frac{\Lambda_{0}}{\Theta_{0}^{2}+\Lambda_{0}^{2}}, \Lambda_{0}=\frac{2 L}{k W_{0}^{2}} \\
\Theta=1+\frac{L}{F}=\frac{\Theta_{0}}{\Theta_{0}^{2}+\Lambda_{0}^{2}}, \Theta_{0}=1-\frac{L}{F_{0}} \\
W_{e}=W\left(1+G_{d}\right)^{1 / 2}
\end{gathered}
$$

On the other hand, regarding the downlink, the following values are substituted into the equation (3).

$$
\begin{aligned}
& G_{d}=4.35 \mu_{2} \Lambda^{5 / 6} k^{7 / 6}\left(H-h_{0}\right)^{5 / 6} \sec ^{11 / 6}(\xi) \\
& \mu_{2}=\int_{h_{0}}^{H} C_{n}^{2}(h) \xi_{0}^{5 / 3} d h, \xi_{0}=\frac{h-h_{0}}{H-h_{0}}
\end{aligned}
$$

With the above-mentioned formulas, we will discuss in the next section with the demand of optical specifications of the optical antenna available for NG-FSO system and RoFSO system.

\section{Fine tracking technology}

The challenge in all-optical connection of FSO and SMF systems is not only to design an effective beam tracking and optical antenna alignment technique, but also an efficient method for focusing the light into the SMF at the receiver. Active tracking is required to maintain alignment of the received optical signal to the SMF. To achieve stable beam spot position control as well as compensate for beam AOA fluctuation caused by atmospheric turbulence we adopted a small size two axis galvanometer type mirror drive mechanism. This mirror device called fine pointing mirror (FPM) is shown in figure 2. The tracking system constitutes the feedback system which considers the direction of the optic axis of a received optical system which changes the arrival directions of beacon light with an input and inclination of FPM as an output. Figure 3 shows a block diagram of a tracking servo system. Although the actual servo system consists of two horizontal independent axes this 


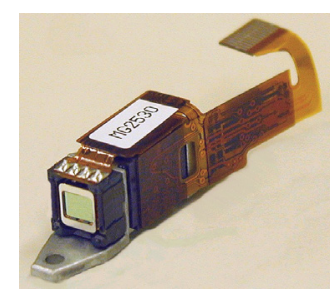

Figure 2. Photograph of FPM

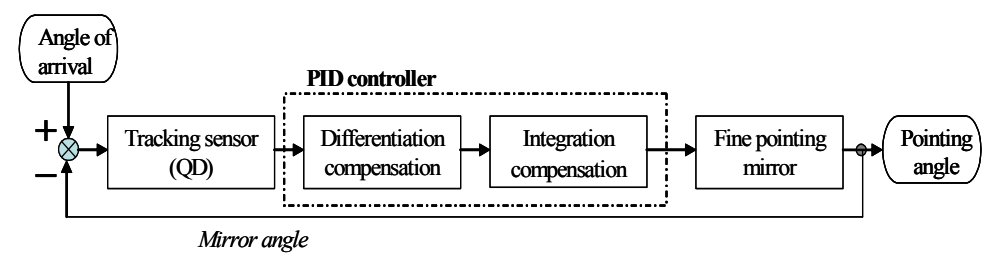

Figure 3. Block diagram of the fine tracking servo system

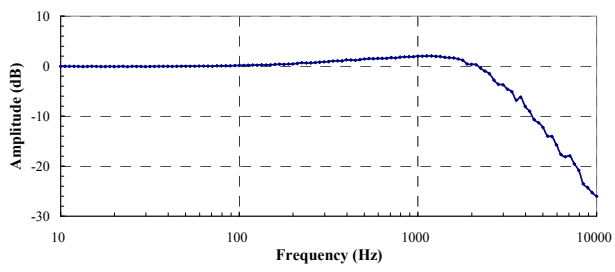

(a) Amplitude

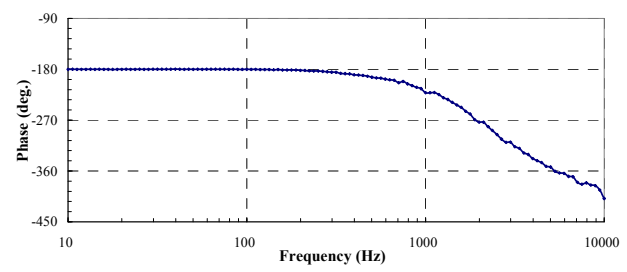

(b) Phase

Figure 4. Frequency characteristics of closed loop feedback system. (a) Amplitude (b) Phase

figure shows only one axis. The position of the received beam is determined by calculation the position of the beacon spot on the four elements of the QD. This signal serves as an input of an analog proportional-integral-derivative (PID) controller. The transfer characteristic of FPM is carrying out the response of a typical secondary system (azimuth (Az) axis is $100 \mathrm{~Hz}$ and elevation (El) axis of resonance frequency is $80 \mathrm{~Hz}$ at the weak oscillating system of dumping), and the PID controller by progress and delay compensation is designed so that a control zone may become the largest in consideration of this characteristic and the nonlinearity of a tracking sensor. As a result, the control zone of the closed loop response has attained the performance of about $2 \mathrm{kHz}$. In the case of a feedback system, disturbance suppression performance is proportional to a closed loop zone. Therefore, this tracking system shows that tracking control more highly efficient a single figure than the conventional tracking system which was an about $100 \mathrm{~Hz}$ control zone was realizable. Disturbance is poured in from the integration input of Figure 4, and the result of having measured the tracking response which appears in a mirror drive output about $\mathrm{Az}$ axis is shown in Figure 5. A vertical axis is the amount of suppression of disturbance amplitude. The characteristic is the same also about $\mathrm{El}$ axis. On the frequency of $100 \mathrm{~Hz}$ or less, it turns out that it has the capability that disturbance can be suppressed or less to 1/100. 


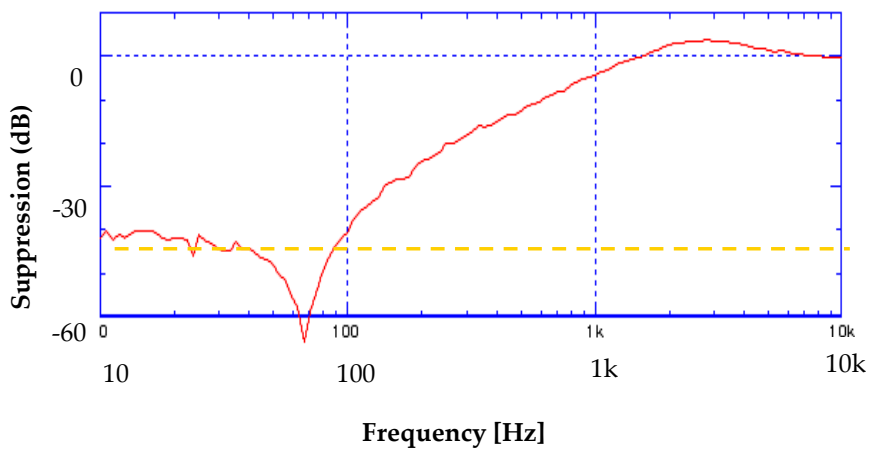

Figure 5. Turbulence suppression characteristics of feedback system

\section{Research and development on NG-FSO system}

\subsection{The basic concept of the NG-FSO}

In research and development of the NG-FSO system, we developed a compact optical antenna suitable for optical communication between satellite in space and deployment in stratospheric platform [Arimoto 2003][Katsuo 2005]. Because of the limitation in payload and equipment size in such usage, a lightweight optical antenna was required designed using off-axis free-form optics without the obscuration [Takahashi 2006]. In general, a Galileo type optical system, composed of a convex lens and a concave lens, is used to convert input/output diameter of laser beam in an optical antenna. However, when using such kind of refractive optical system it is difficult to suppress reflection of each surface of lenses in a wide wavelength range, so measurement errors as a result of ghost and/or flare can arise. In addition, since this system is accompanied with a chromatic aberration, adjustment of the focal length based on a wavelength is necessary. Therefore, a reflection based optical system which does not have a chromatic aberration is suitable for an optical system that is incorporated into a highly precise optical antenna. Although there are many cases that a Cassegrain type configuration is used for beam expander of reflecting mirror system, it is not suitable for the optics of the antenna of this purpose because of an obscuration in the beam center caused by secondary mirror. We abandoned the constitution on co-axial optical system and devised off-axial reflection optical system instead. In case of optical wireless communication on ground or between ground and space, the fluctuation of arrival beam angle is caused by the atmospheric turbulence. Therefore, if comatic aberration of the optical antenna is large, beam expander causes peculiar aberrations according to an incident angle of laser beam. Because rotational asymmetric aberration (shown in Figure 6(b)) by the decentering of the optical element occurs as well as radial aberration such as the comatic aberration, in the case of an off-axial optical system, all those aberration becomes able to be compensated by using free-form surface for all mirrors. The free-form surface [Takahashi 2011] is curved surface defined in XY polynomial such as equation 13. 


$$
Z=\sum_{n=0}^{k} \sum_{m=0}^{n} C_{n m} X^{m} Y^{n-m}
$$

In one aspect, free-form surface becomes able to have positive and negative power (Figure 6(a)). Furthermore, the curvature of the tangential direction can be different from azimuth direction.

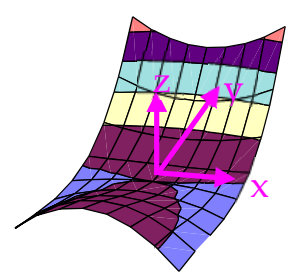

(a) Example of a free-form surface

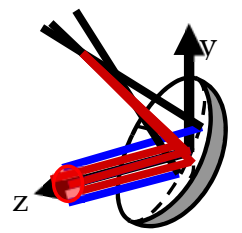

Axial comatic aberration

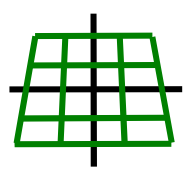

Trapezoid

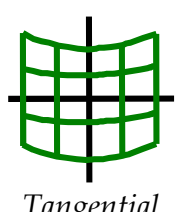

Tangential

Asymmetrical Distortion

Figure 6. A free-form surface and Asymmetrical aberrations

\subsection{Consideration of the optical specifications}

The average expansion and fluctuation of light intensity of the laser beam at $1.550 \mu \mathrm{m}$ wavelength with the atmospheric turbulence are calculated by using the expressions of Section 3 stated above. In this calculation, the transmission beam is Gaussian beam which have beam divergence of diffractive limit, the difference in altitude between the two stations (low altitude stratospheric platform experiment [Katsuo 2005] condition) is $4 \mathrm{~km}$, zenith angle is 60 degree, the transmission distance of the beam is $8 \mathrm{~km}$ maximum, and the pointing errors is $0 \mu$ radian. When the transmission beam radius is $40 \mathrm{~mm}$, the free space loss is approximately $1 \mathrm{~dB}$. So that if the optical antenna achieves an output transmission of around $100 \mathrm{~mW}$ and the receiver sensitivity of $-20 \mathrm{~dB}$, we can expect a margin of $40 \mathrm{~dB}$ and it is possible to communicate under thin cloud or fog conditions. The relation between the beam radius at the transmitter (W0) and the average beam expansion at receiver point is shown in Figure 7. The mean intensity fluctuation of the received beam under similar conditions is shown in Figure 8. According to these results, the radius of received beam is varied slightly and the expansion rate of the radius of received beam is constant when the radius of transmit beam is more than $60 \mathrm{~mm}$. Moreover, the larger the transmission beam diameter is, the smaller the amount of the decrease of center strength of the received beam, and the free space transmission loss is less than $5 \mathrm{~dB}$ for a transmit beam diameter of uplink $70 \mathrm{~mm}$ or downlink $50 \mathrm{~mm}$ or more. Assuming $\mathrm{Cn}^{2}$ is $1.7 \times 10^{-14}(\mathrm{H} / \mathrm{V}$ model $)$, we calculated the fluctuation of the received light intensity (scintillation index) by varying the transmitted beam radius. The result is shown in figure 9 .

The change is small in weak fluctuation case and there is no big received optical power changes for propagation distance of $\sim 4 \mathrm{~km}$ with laser beam transmit and receive aperture diameter of $10 \sim 100 \mathrm{~mm}$ therefore an optical antenna having this range of aperture beam 
transmission is possible. Because this fluctuation is significantly small and in the region of weak fluctuation and there is not large fluctuation of intensity of the received beam, the optical antenna which has such diameter of aperture transmits the optical beam have the possibility. The calculation result of receive light intensity fluctuation when the transmit beam radius is set at $20 \mathrm{~mm}$ and pointing error is $1 \mu$ radian are shown in figure 10 . On the assumption that the inoperable rate is $10^{-7}$, it is necessary to consider the fading margins which are $3.6 \mathrm{~dB}$ for the uplink and $5.3 \mathrm{~dB}$ for the downlink. From these calculations, it is desirable for the pointing error to be around $1 \mu$ radian in consideration of fading when a diffraction limited Gaussian beam is transmitted.

The pointing error of the FPM used for the fine tracking of the NG-FSO system in this research is estimated to be approximately 20 uradian. The angular magnification of the optical antenna is 20, and the tracking accuracy in the system can expect the improvement of the atmosphere fluctuation to become 1.0 Mradian in a calculation. Considering the above outlined analytical results, the target specifications of the optical system are as follows:

1. Optical antenna aperture diameter is $40 \mathrm{~mm}$

2. Angular magnification is 20

3. Coupling efficiency with SMF on axis is around $-1 \mathrm{~dB}$

4. Resolution is diffraction limit of field angle \pm 0.2 degrees (less than wave aberration $\lambda / 10)$

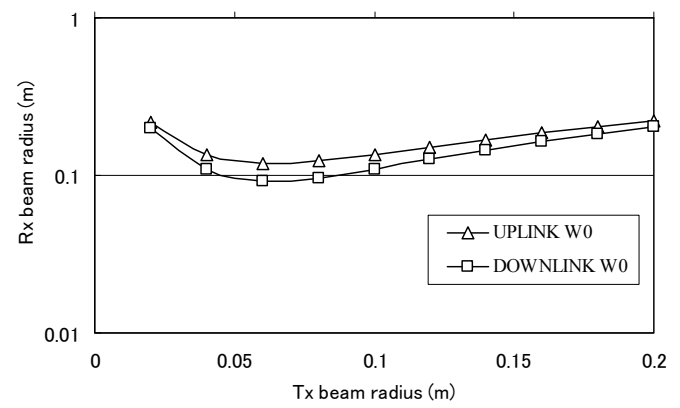

Figure 7. Rx beam radius versus $\mathrm{Tx}$ beam radius

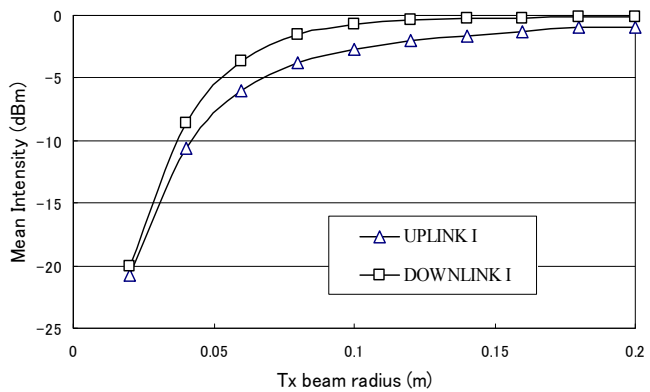

Figure 8. Mean intensity depend on Tx beam radius 


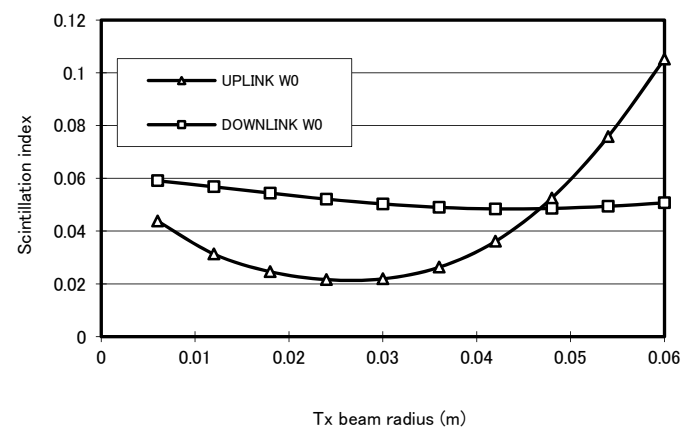

Figure 9. Scintillation index depend on Tx beam radius

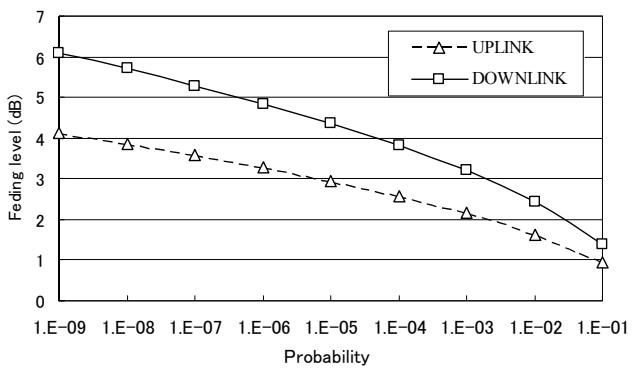

Figure 10. Probability of fading level

\subsection{Result of optical design}

In order to conduct an optical system without obscuration, having less deterioration of wavefront accuracy when the incident beam angle varies, we designed an optimum optical system comprising off-axial free form surface triple mirror which have enlargement/reduction rate of 20 times. Optical layout of triple mirror is shown in figure 7. Fundamental power placement in an optical design is a primary concave mirror plus a secondary convex mirror. With this optical configuration, a primary image is formed whose spherical and coma aberrations are compensated, and by using a collimator mirror which is arranged after the primary image a beam of parallel rays of light is producing. We set the paraxial focal length of the primary mirror and the secondary mirror at $f_{1}=230 \mathrm{~mm}$, the collimator mirror at $f_{2}=11.8$ $\mathrm{mm}$, and the angular magnification of the triple mirror at approximately 20. Moreover, we have decentered aberrations, such as axial comatic aberration and axial astigmatism caused by tilt and decentered of both reflective surfaces, compensated by the three pieces of free-form mirror. A figure of constitution of the prototype of the optical antenna module which had a QD feedback type built-in fine tracking system by a microminiaturized fine pointing mirror (FPM) is shown in figure 11, the photograph is shown in figure 12, and the photograph is shown in figure 13. Based on the design result, the calculations of coupling efficiency when an ideal lens is deployed in the radiation side are shown in figure 14. 


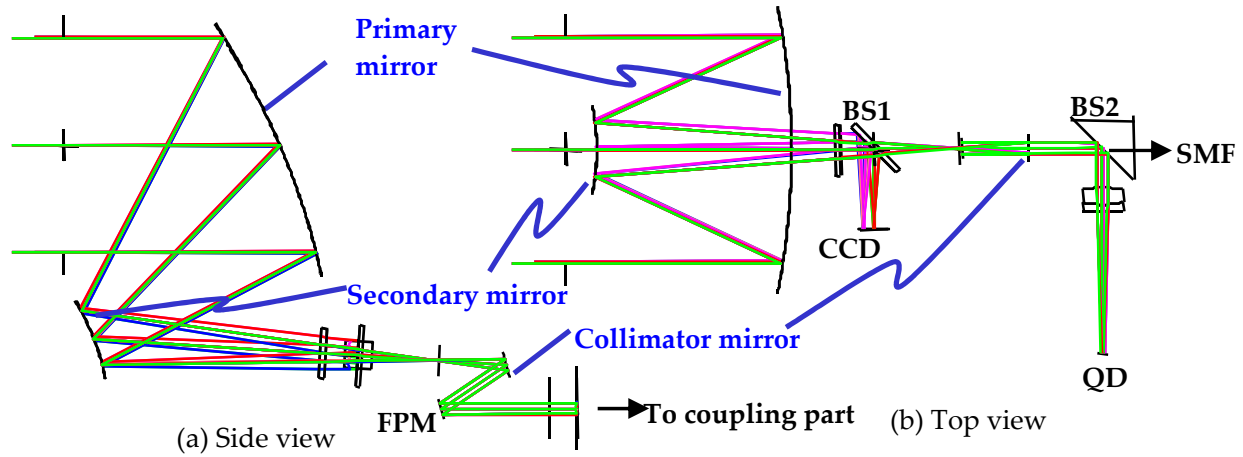

Figure 11. Optical layout of an optical antenna with off-axial free form optics.

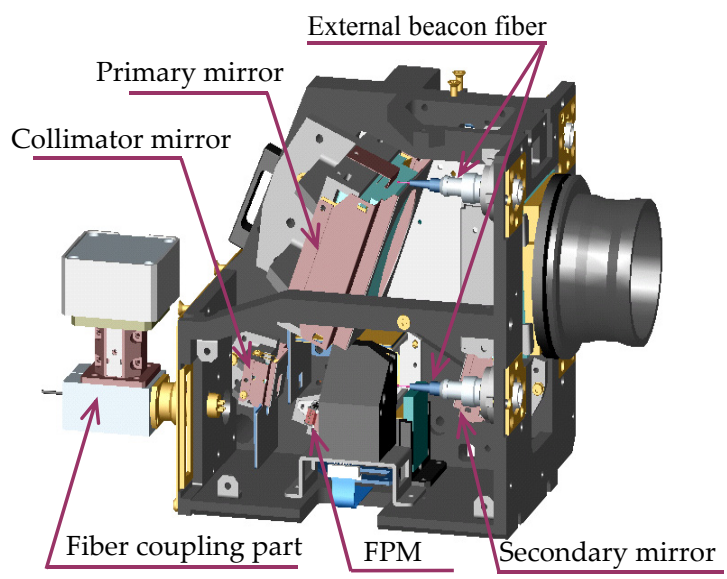

Figure 12. Appearance of prototype of optical antenna module

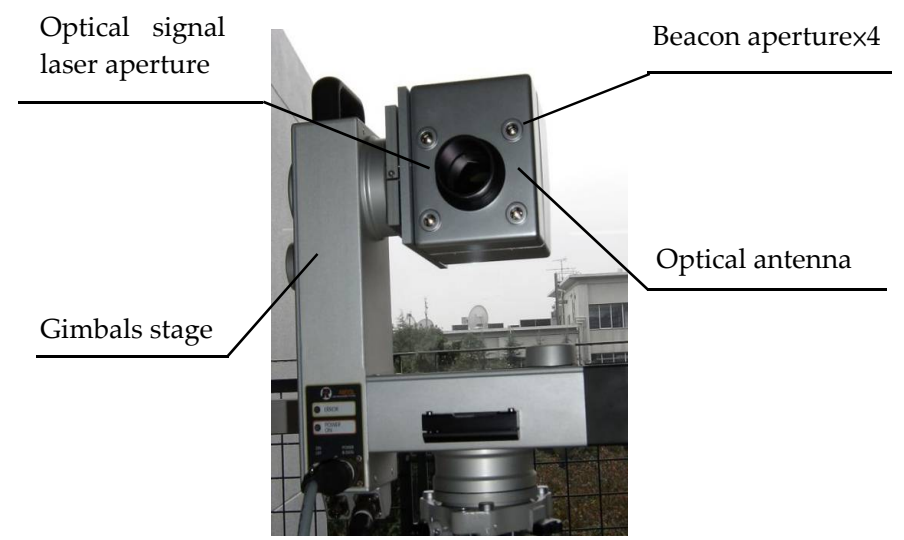

Figure 13. Photograph of prototype optical antenna on the gimbal stage 


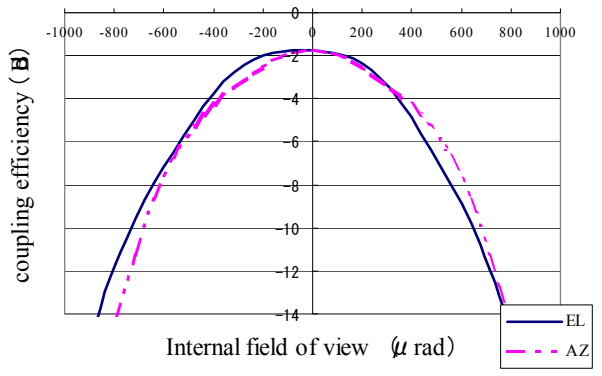

Figure 14. Coupling efficiency depend on internal field of view

\subsection{Experimental set up of NG-FSO system}

The test beds were put on two campuses of the Waseda University campus area situated in Shinjuku Ward, Tokyo city [Kazaura 2007][Kazaura 2006]. Figure 11 shows a map view of where the FSO systems are deployed. Two antennas were set on the rooftop of a 10 floor building (figure 15) and the corresponding antennas on the rooftop of a 9 floor building with a link spanning $1 \mathrm{~km}$ between the transceivers on the top of those two buildings. The antenna shown on the right in figure 16 is the antenna under experiment while the antenna on the left is used for scintillation and optical power attenuation measurement. They are both connected to data acquisition system placed in the experiment setup room which records all the data as shown in figure 17. The FSO beam propagation path travels over low rising residential, office or campus buildings, thus transmitted beam will experience higher probability of scintillation from ground heating and heating from these buildings. The experimental antennas use a $980 \mathrm{~nm}$ beacon for alignment and $1550 \mathrm{~nm}$ wavelength for communication. A charge coupled device (CCD) camera is used for initial alignment purposes which is done manually adjusting thimble screws located at the base of the antenna. Repeated adjustments are made on both sides until the light is centered on both CCD cameras (see TV monitor in figure16). Fine alignment of the antennas is achieved by using a QD which gets a feedback of the maximum received power from the inbuilt data acquisition system. The $1550 \mathrm{~nm}$ transmission beam was driven by an erbium doped fiber amplifier (EDFA) capable of outputting $100 \mathrm{~mW}$ total power. The experimental antenna can be controlled from a remote location by a serial computer interface to send control information that is used for remote alignment. For the scintillation and optical power attenuation measurement antennas, a serial computer interface provides the beam output power and receives signal strength information and other information.

The basic configuration of our experimental setup for the developed FSO system by alloptical connection of free-space and SMF is show in Figure 18. Because the signal light is fiber coupled at both ends, booster/post EDFA and other measurement and data collection devices can be conveniently placed inside the building. The fibers are run to the respective rooftops and then coupled directly to the antenna as shown in Figure 16. Separation of transmitting light and receiving light is performed by an optical circulator. The test signal 


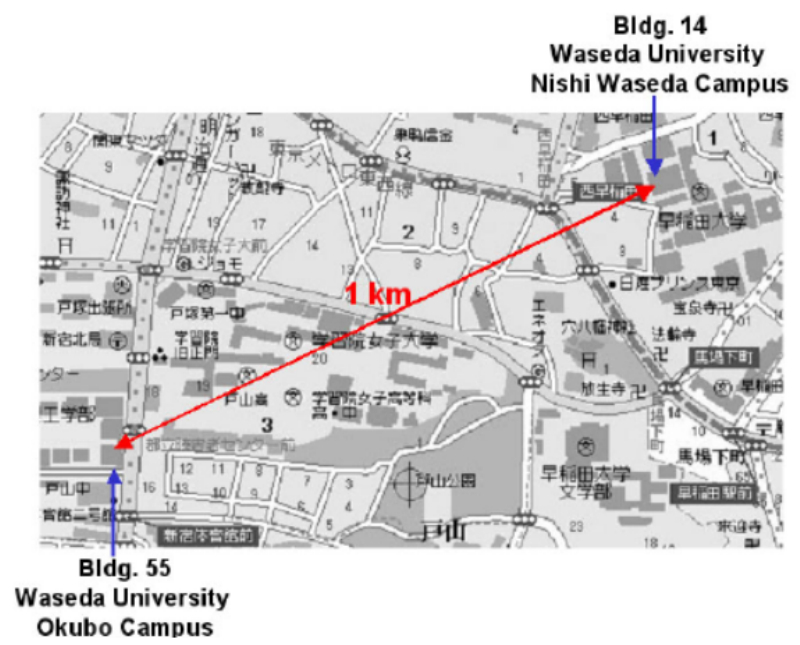

Figure 15. A map view of the $1 \mathrm{~km}$ test between Waseda University Nishi-Waseda campus and Okubo campus

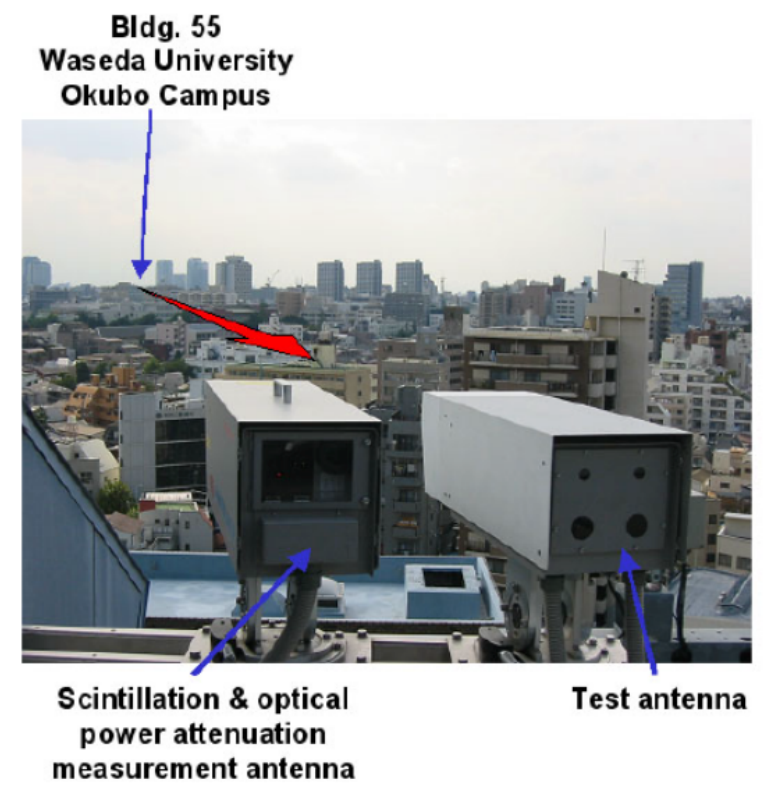

Figure 16. Optical antennas on the rooftop of building 14 in Nishi-Waseda

(pseudo random bit sequence) from bit error rate tester (BERT) is changed into a light signal by an E/O converter, and after being amplified with a booster EDFA, it is sent to the optical antenna of the rooftop. At the other end after the optical signal received with the optical antenna is removed in amplification by post-EDFA and a light filter removes noise, it is changed into an electric signal by an $\mathrm{O} / \mathrm{E}$ converter, and becomes a receiving bit sequence in 
a clock data recovery circuit, and calculation of the bit error is carried out by BERT. The light signal which branched by $3 \mathrm{~dB}$-coupler on the way is used for the monitor of receiving intensity, or other measurement. In a WDM experiment, the wavelength of each signal is made to fit the ITU grid (100 GHz channel spacing), and multi/de-multiplex is performed using a DWDM multi/de-multiplex device. Moreover, a weather monitor device and another optical antenna are installed in the rooftop and weather condition (visibility, precipitation and temperature) and link line condition (scintillation and optical power attenuation) are measured simultaneously.

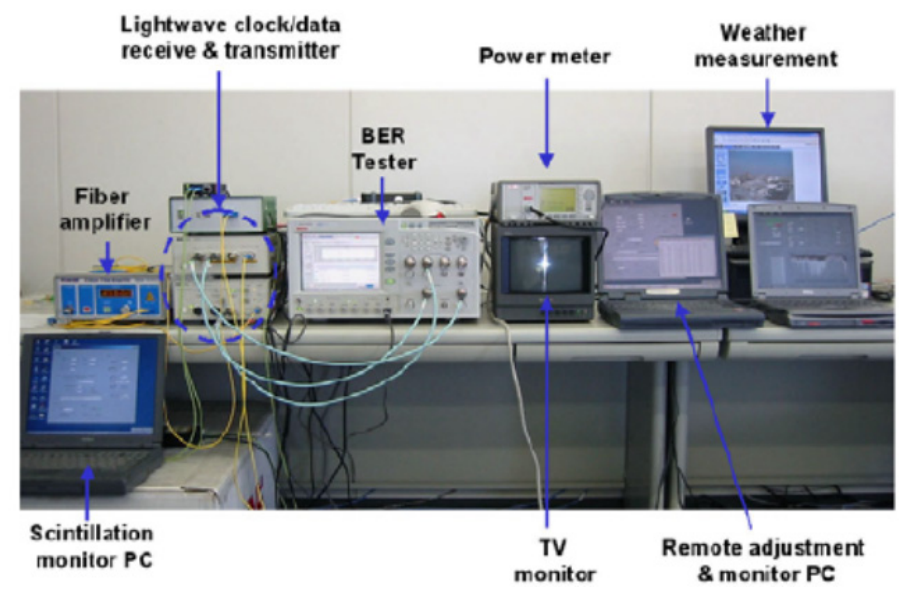

Figure 17. Photograph of experimental hardware setup

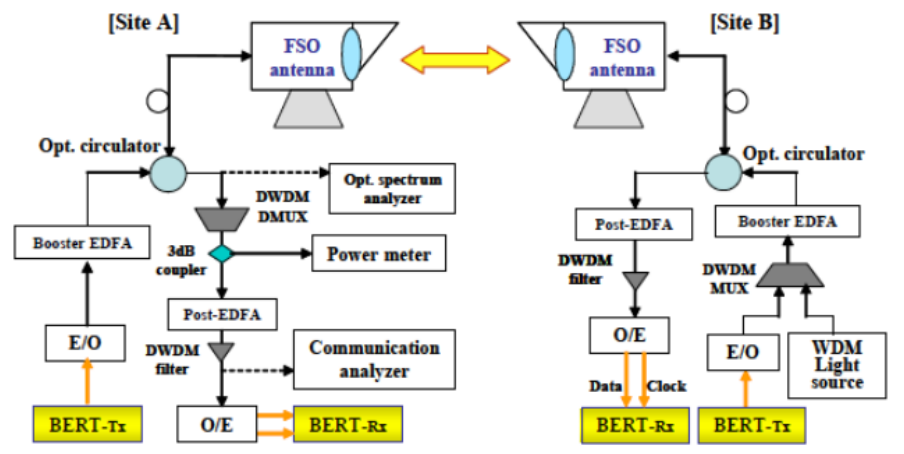

Figure 18. Experimental FSO communication system setup

\subsection{Result and analysis}

First, in order to evaluate the all-optical connection FSO system characteristic, the result of the 10 Gbps single wavelength light signal transmission experiment [Arimoto 2007] which is not achievable with conventional FSO system is shown in Figure 19. The bit error rate (BER) is measured by accumulated at 1 minute interval is shown in the figure. The data encoded 
optical signal, is amplified by $100 \mathrm{~mW}$ booster EDFA shown in Figure 18. The minimum receiving sensitivity of the optical receiver used in this experiment is $-37 \mathrm{dBm}$. Stable transmission with few errors has been achieved over 12 hours. Figure 20 shows the eye patterns measured continuously for 5 minutes at this time. Although the fluctuation of the amplitude direction becomes large when compared with the eye patterns before transmission, it seems that there are very few increases in a time jitter (horizontal axis), and high-speed transmission is possible. In addition, the error sometimes shown in Figure 19 could be burst error produced by the momentary fall of received light intensity of several milliseconds or less, and this influence can hardly be observed on TCP/IP which is commonly used. An experiment using two optical wavelengths and connection to a Giga-bit Ethernet circuit and a $2.5 \mathrm{Gbps}$ test signal was conducted. Optical and electrical signal conversion were performed by the media converter, and the Ethernet circuit performed huge size file transfer of between PCs connected through a switch, and has calculated the throughput from transfer time. In addition, it is connection by $100 \mathrm{Mbps}$ between the switch and PC. Figure 21 shows the measurement result of the throughput of the file transfer performed for every minute and BER accumulated per minute. The minimum receiving sensitivity is $-34 \mathrm{dBm}$ which is almost same as the optical receiving module currently used for the media converter and the optical receiver currently used for BER measurement. Although errors sometime appeared in BER measurement, there is almost no change of a throughput almost similar result as in wired network. Since the great portion of error is short-time burst error, the throughput is hardly affected. Moreover, to the experimental result of BER and Ethernet, change considered to be mutual interference among other light signals cannot be found out at all, but has realized stable WDM transmission to it.

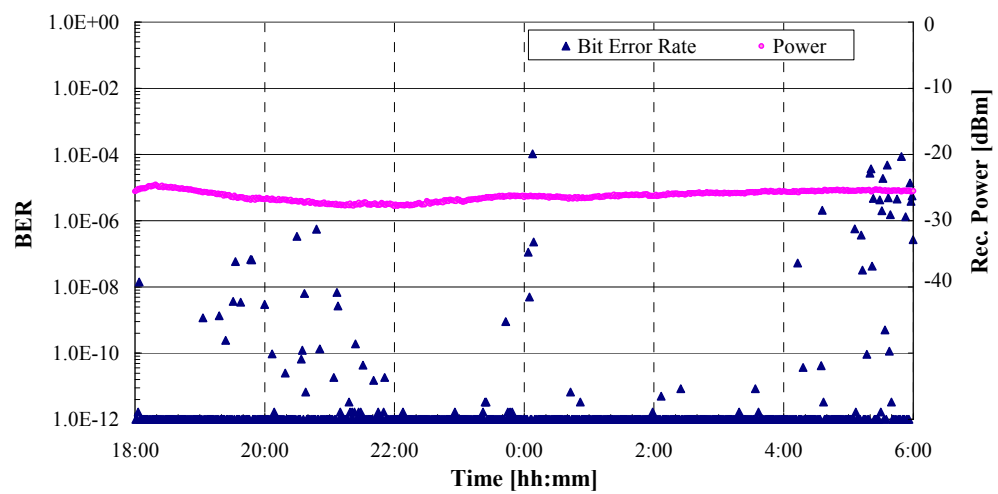

Figure 19. BER measurement result for $10 \mathrm{Gbps}$ transmission over $1 \mathrm{~km}$ distance.

From these results, we demonstrated the next-generation FSO system using all-optical connection technology realized $10 \mathrm{Gbps}$ transmission and also possible to apply WDM transmission which were not able to be realized in the conventional FSO system, and showed that the capability near an optical fiber could be offered. However in presence of strong atmospheric turbulence increased burst errors are observed in the system because of 
the fall in the received signal power. In order to realize stable communication even under strong atmospheric turbulence more improvement in is needed is required in the tracking system performance. Moreover, it is important to clarify the link design technique about the application distance of atmospheric turbulence and a FSO system.

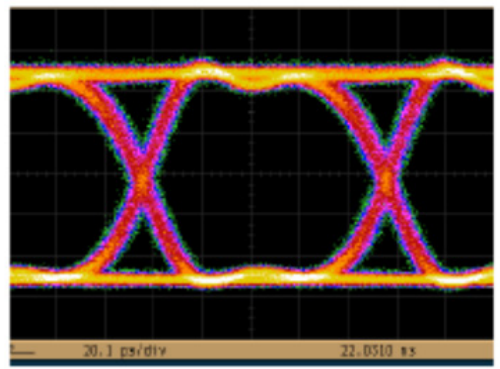

(a)

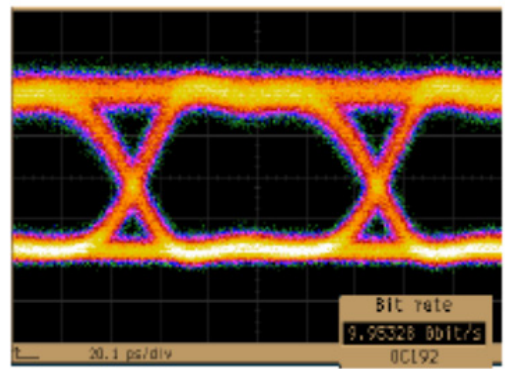

(b)

Figure 20. $10 \mathrm{Gbps}$ transmission test eye pattern. (a) Before transmission (b) After $1 \mathrm{~km}$ transmission

\subsection{Conclusions of the NG-FSO system}

The next-generation optical wireless communication system offered seamless connection of free space and fiber system. The transceiver incorporates a FPM for high-speed beam tracking and control function, therefore, having the capability to mitigate the effects of atmospheric turbulence on the transmitted optical beam. The FSO system performance was verified and error free transmission over an extended period of time was demonstrated. The system performance expressed in terms of BER performance was also evaluated and showed to be consistently above acceptable levels. Stable performance after increasing the system bandwidth using WDM technology was also attained.

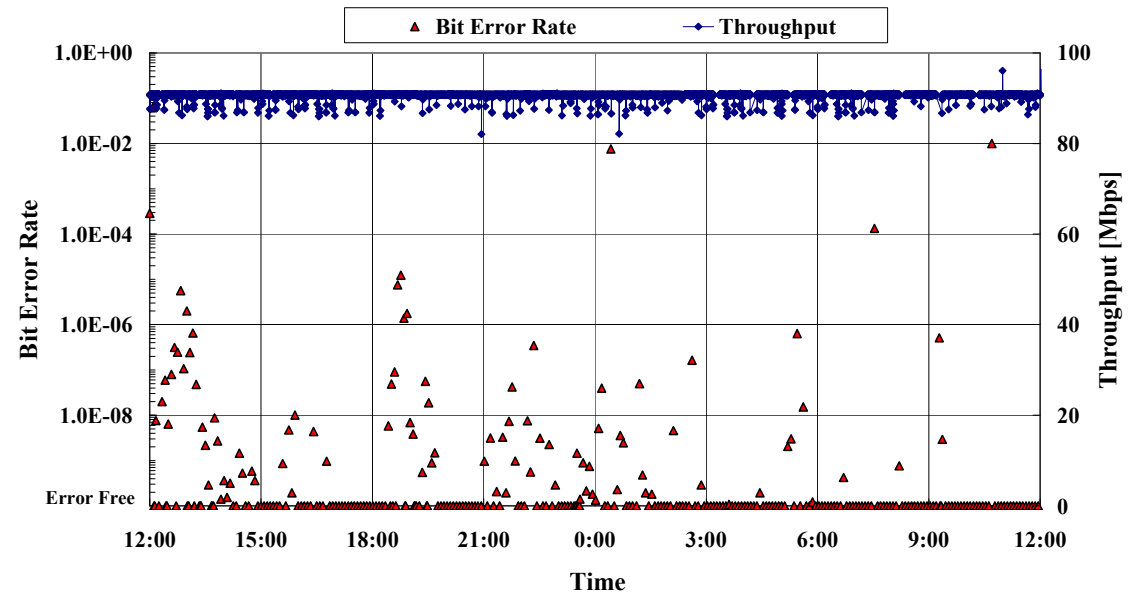

Figure 21. Throughput and BER characteristics in DWDM transmission 


\section{Research and development on RoFSO system}

\subsection{Description of RoFSO system}

Examination of a RoFSO system which expands RoF technology to a free-space using alloptical connection technology is shown. RoF technologies [Al-Raweshidy 2002][Hai 2006] can realize a cost effective universal platforms for future ubiquitous wireless services. Furthermore, RoF networks can be extended to virtual radio free-space network with layer 1 routing realized [Komaki 2003][Tsukamoto 2005]. By using RoF, architecture for radio access zones easily employs micro or pico cellular systems. However, the optical fiber as an infrastructure is needed for a RoF network. We aim at quick and effective provide of heterogeneous wireless services for not only urban area but rural area, that has a little or no infrastructure for broadband services by the system with which developed next-generation FSO and a RoF system were united.

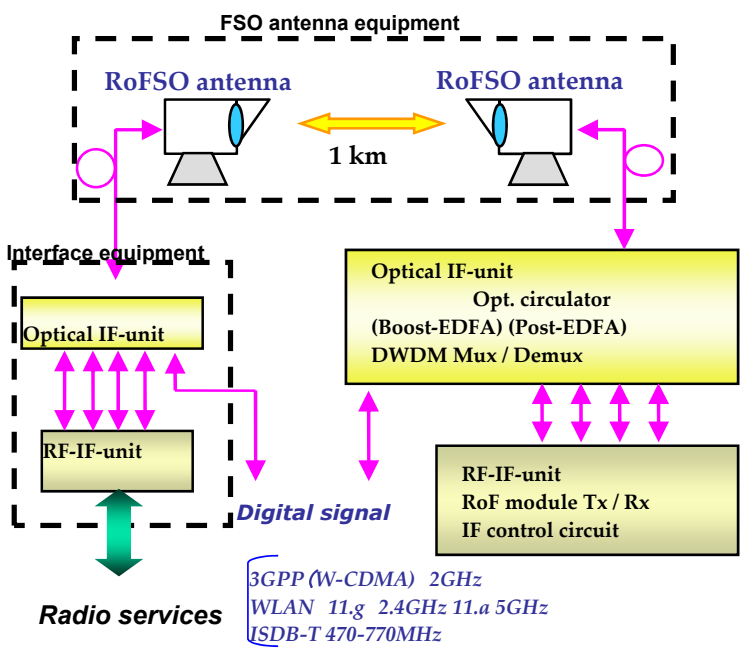

Figure 22. Outline of RoFSO system setup

We developed the RoFSO antenna which is improved the optical antenna, and installed in the same experimental field [Kazaura 2009][ Kim 2009][Tsukamoto 2008]. Moreover, RF interface which represents the RoF system, and optical interface which performs multi/demultiplex and amplification of the optical signal carrying the various services developed for an experiment as indoor equipment. A new optical antenna of $80 \mathrm{~mm}$ of the main aperture aimed at better tracking accuracy by feeding back the influence of the atmospheric fluctuation which signal light receives itself by diverting and using a part of signal light for fine tracking. RF interface unit consists of a RoF module which performs the electro/optical conversion of RF signal corresponding to the planned service (3G cellular, WLAN 11g/a, DBV-T), and its control circuit. Each light signal transmitted is matched with the ITU grid wavelength of a $100 \mathrm{GHz}$ space. The optical interface unit consists of a DWDM multi/de- 
multiplex device, EDFA (booster/post) and optical circulator for separation of transmit/receive signal. A figure showing the whole system just described is depicted in Figure 22. In this experiment, signal generators and analyzers for evaluating the transmission quality of various services are connected to RF interface unit.

\subsection{Consideration of optical antenna specification}

The average expansion and fluctuation of light intensity of the laser beam at $1.550 \mu \mathrm{m}$ wavelength with the atmospheric turbulence are calculated by using the above expressions (Section 3). The relation between the beam radius at the transmitter and the average beam expansion at receiver point is shown in Figure 23.

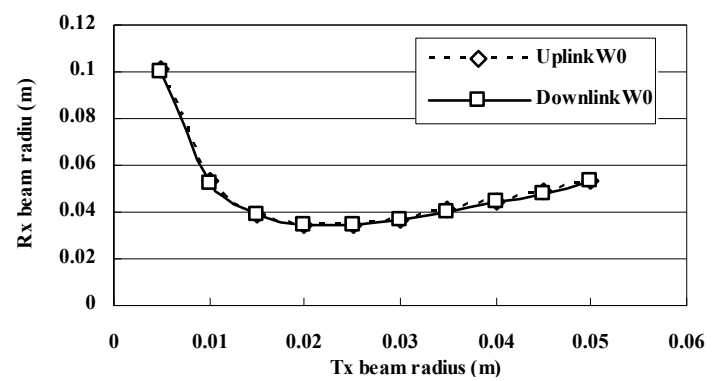

Figure 23. $\mathrm{Rx}$ beam radius versus $\mathrm{Tx}$ beam radius

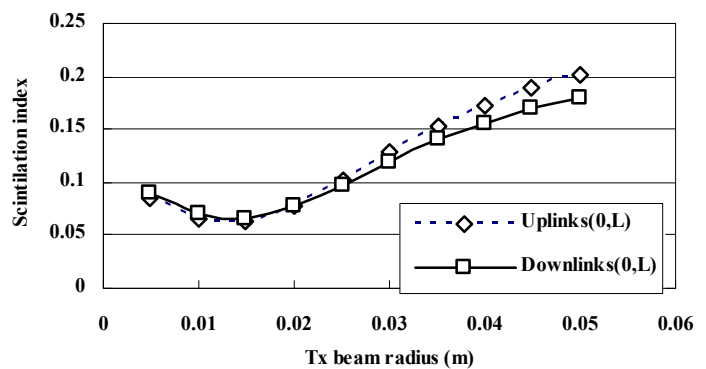

Figure 24. Scintillation index depend on Tx beam radius

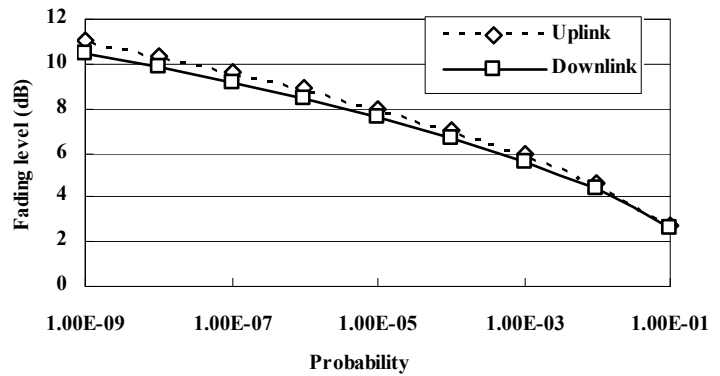

Figure 25. Probability of fading level 
According to these results, the radius of received beam is varied slightly and the expansion rate of the radius of received beam is constant when the radius of transmit beam is more than $20 \mathrm{~mm}$. Moreover, the larger the transmission beam diameter is, the smaller the amount of the decrease of center strength of the received beam, and the free space transmission loss is less than $5 \mathrm{~dB}$ for a transmit beam diameter of $40 \mathrm{~mm}$ or more. We calculated the fluctuation of the received light intensity (scintillation index) by varying the transmitted beam radius. The result is shown in figure 24 . The change is small in weak fluctuation case and there is no big received optical power changes for propagation distance of $1 \sim 2 \mathrm{~km}$ with laser beam transmit and receive aperture diameter of 10 100 $\mathrm{mm}$ therefore an optical antenna having this range of aperture beam transmission is possible. Figure 25 shows the cumulative probability distribution when the transmit beam radius is set at 40 $\mathrm{mm}$ and pointing error is 5 uradian. We found that the fluctuation of receiving optical intensity is $\pm 3 \mathrm{~dB}$ at $10 \%$ and $\pm 8 \mathrm{~dB}$ at $0.001 \%$. In other words, it is necessary to consider fading margins that are $9.6 \mathrm{~dB}$ for the uplink and $9.2 \mathrm{~dB}$ for the downlink if inoperable ratio is $10^{-7}$.

In addition, the previous NG-FSO system, the beam for fine tracking systems was a beacon light which was different from the signal light. In that case, the propagation path of the beacon light signal and signal beam is not exactly consistent therefore the atmospheric fluctuation behavior is also not similar. Especially, in the case of the data rate is high, such tendency is significantly. Therefore, it was not possible to get enough fine tracking characteristics. That is why in the new system for fine tracking the signal light is used for detection of angle of arrival by utilizing beam splitter.

Based on these calculations, if a diffraction-limited Gaussian beam is transmitted, it is desirable for the pointing error to be less than $5 \mu$ radian considering fading. The pointing error of the FPM used for the fine tracking of the RoFSO system is approximately $20 \mu$ radian and the angular magnification of the optical antenna is 40 . The tracking accuracy of the entire system can be calculated by the division of those two values and it is $0.5 \mu$ radian. Thus, it is possible to expect the improvement of the atmosphere fluctuation.

\subsection{Results of optical design}

A constitution of the prototype of the optical antenna module [Takahashi 2008] which had a QD feedback type built-in fine tracking system by a micro-miniaturized fine pointing mirror with the part of SMF coupling components is shown in figure 26, the photograph of SMF coupling part and tracking system is shown in figure 27 and the photograph of entire prototype optical system is shown in figure 28. Based on this design, results of the calculation of coupling efficiency and spot diagram when an ideal lens is deployed in the radiation side are shown in figure 29. The on-axis SMF coupling loss is $-1.02 \mathrm{~dB}$ with less the $-5 \mathrm{~dB}$ loss for up to full size field angle $25 \mu$ radian. Optical performance for on-axis and offaxis of the designed optical system is summarized in table 1. It is observed that it is possible to obtain almost twice image formation efficiency of the diffraction limit of the design requirement for field angle \pm 0.5 degrees. 


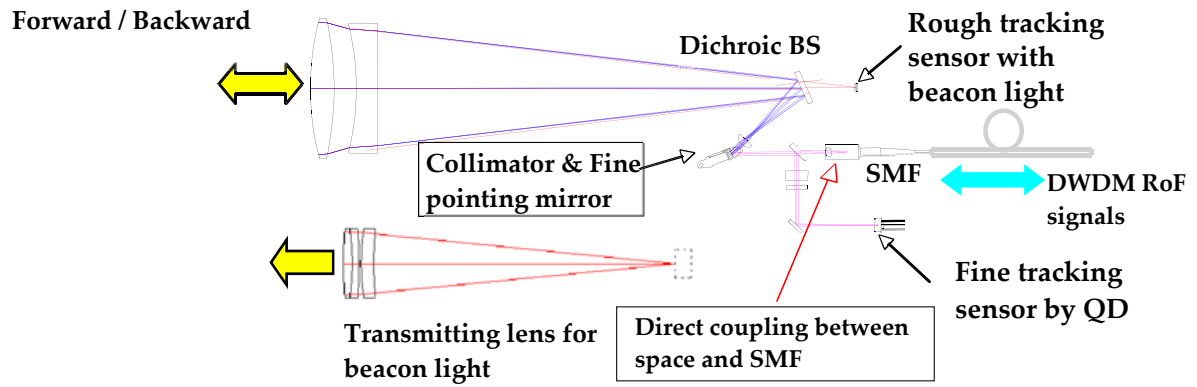

Figure 26. Optical layout of the antenna of RoFSO system

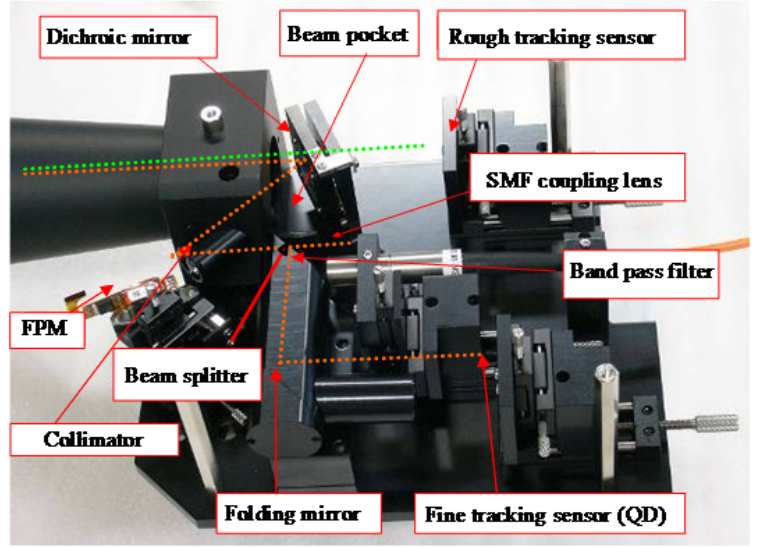

Figure 27. Photograph of SMF coupling part and tracking system

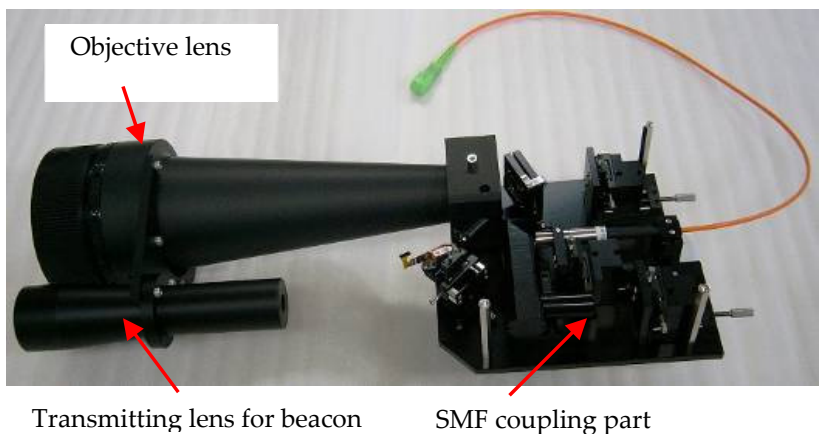

Figure 28. Photograph of entire optical antenna of prototype RoFSO system

To evaluate real performance in the trial product; the coupling efficiency that measured in experiment system deploy a coupling lens after an object lens and a collimator, and to be received light to SMF is approximately $-5 \mathrm{~dB}$. 


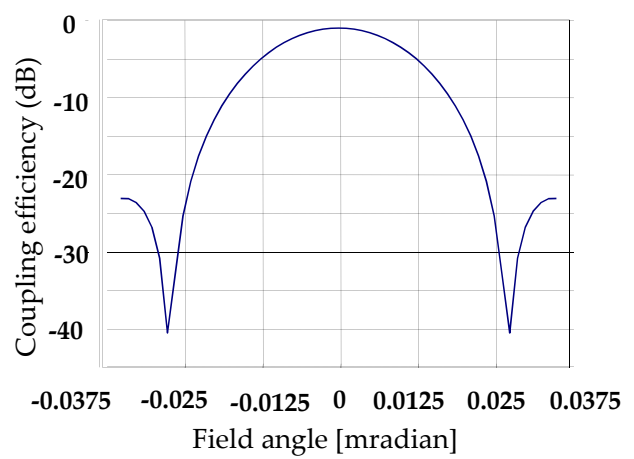

(a) Coupling efficiency

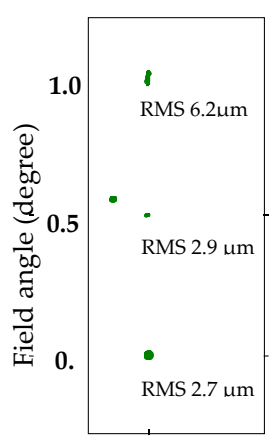

(b) Spot diagram (RMS)

Figure 29. RoFSO antenna optical performance (a) Coupling efficiency (b) Spot diagram

\begin{tabular}{|l||l|l|l|}
\hline Field angle $(\mathrm{deg})$ & 0 & 0.5 & 1.0 \\
\hline \hline Spot diameter $(\mu \mathrm{m})$ & 2.7 & 2.9 & 6.2 \\
\hline Wave aberration $(\lambda)$ & 0.0037 & 0.0094 & 0.0327 \\
\hline Coupling efficiency $(\mathrm{dB})$ & -1.02 & - & - \\
\hline
\end{tabular}

Table 1. Optical performance of the RoFSO optical antenna in design condition

\subsection{Evaluation of prototype RoFSO system}

\subsubsection{Experimental evaluation setup}

We installed the developed RoFSO system at a same place of NG-FSO system in Waseda University and performed experiments [Kazaura 2009] [Kim 2009] [Tsukamoto 2008] [Takahashi 2008]. Photographs showing the devices setup on the rooftop as well as the various measurement devices setup in the laboratory is depicted in Figure 30(a) and 30(b) respectively. Three FSO antennas are placed on the rooftop of one building as shown in Figure 30(a), which include the DWDM RoFSO antenna under investigation and two other conventional antennas used for measuring and quantifying the deployment environment characteristics for example atmospheric turbulence induced scintillation.

\subsubsection{Basic performance}

As a result, the system's stability and tracking properties satisfied the design requirements (tracking response is more than $2 \mathrm{kHz}$, turbulence suppression characteristics of this feedback system are more than suppression ability $20 \mathrm{~dB}$ in frequency less than $100 \mathrm{~Hz}$ with $\mathrm{Cn}^{2}<1.7 \times 10^{-14}$ ), and we confirmed that the system can transmit WDM with sufficient performance regarding not only error-free transmission of digital signals but also RF signal transmission. Figure 31 shows the intensity fluctuation characteristics of received beam 
when the fine tracking of antenna is set $\mathrm{OFF}$ or $\mathrm{ON}$, and the average of turbulence $\mathrm{Cn}^{2}$ is $2 \times 10^{-14}$. If the fine tracking is set $\mathrm{OFF}$, the fluctuation of receiving optical intensity largely and frequently decays because it is not possible to control arrival angle, also because location of beam spot of SMF consistently fluctuates. In addition, the mean of receiving optical intensity is low because it is not possible to control location of light focus accurately. On the other hand, the fine tracking of antenna is set to $\mathrm{ON}$, the entire fluctuation decreases and the mean of receiving optical intensity is improved. Figure 32 shows the receiving optical spectrum when four of wireless service signals are transmitted with $1 \mathrm{~km}$ distance and WDM transmission using this RoFSO. It can be found that each service signal is clearly separated and is transmitted without mutual interaction.

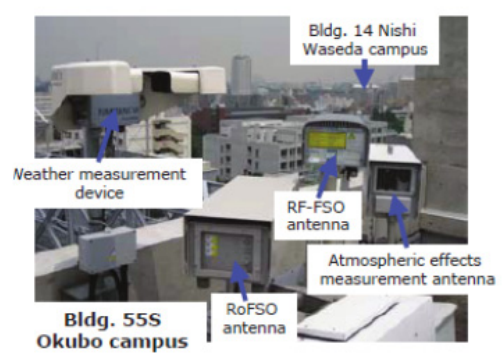

(a)

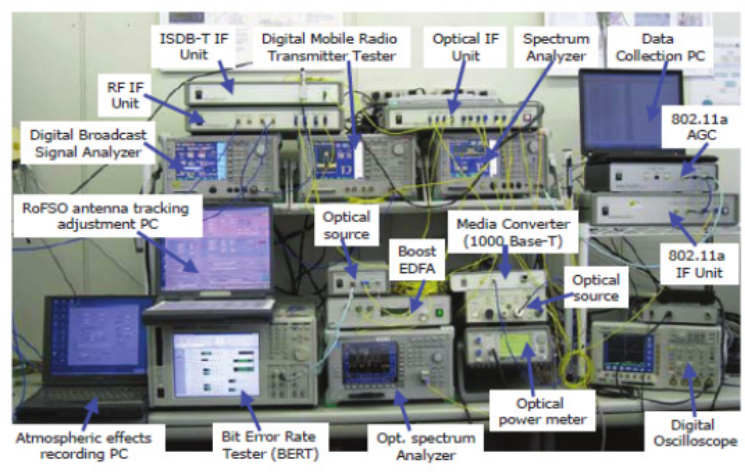

(b)

Figure 30. RoFSO system experimental device (a) rooftop setup and (b) devices setup in the laboratory

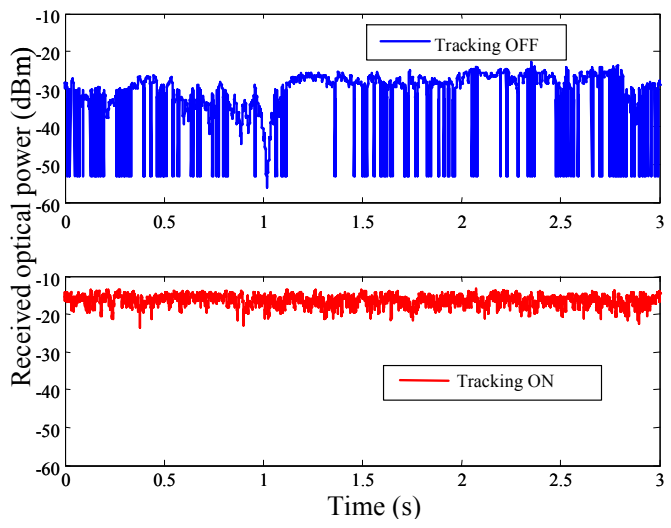

Figure 31. Optical intensity fluctuation characteristics of received beam with tracking OFF (upper) and tracking ON (lower) 


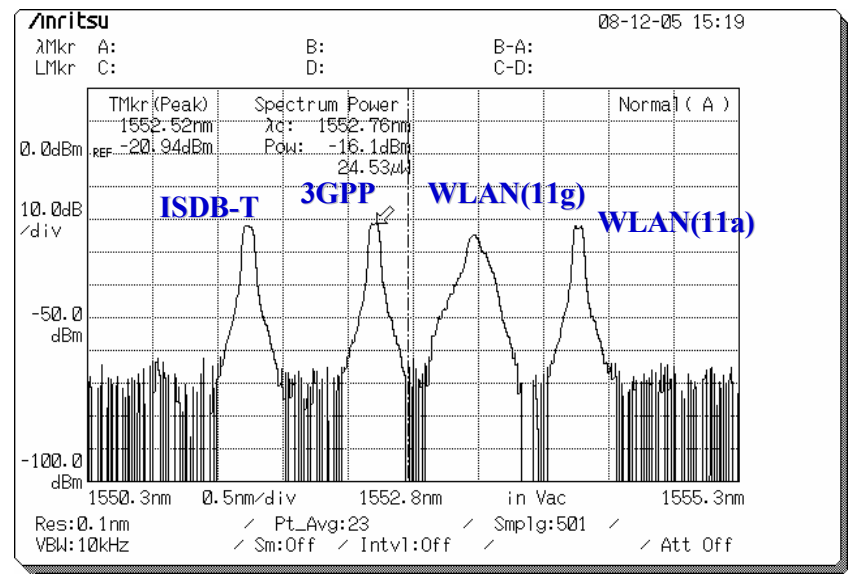

Figure 32. Optical spectrum of four wireless service signals using WDM RoFSO system

\subsubsection{W-CDMA (Cellarer phone)}

In W-CDMA system, the downlink signal transmitted by the base station is designed to fulfill the specifications set in 3GPP standard [3FPP 2002]. The spectral properties of the signal are measured by he adjacent channel leakage ratio (ACLR) which is considered to be a more stringent quality metric parameter, and is defined as the ratio of the amount of leakage power in an adjacent channel to the total transmitted power in the main channel. The 3GPP specifies one main channel and two adjacent channels. The standard requires the ACLR to be better than $45 \mathrm{~dB}$ at $5 \mathrm{MHz}$ offset and $50 \mathrm{~dB}$ at $10 \mathrm{MHz}$ offset. In our experimental setup, we use a signal generator to generate a test signal (W-CDMA Test Model 1) with a signal power of $-20 \mathrm{dBm}$ which is transmitted over the RoFSO link and at the receiver side a digital mobile radio transmission tester is used to measure and record the quality of the W-CDMA signal. Figure 33(a) shows a received W-CDMA signal ACLR spectrum after transmission over the $1 \mathrm{~km}$ RoFSO link. It is observed that the signals' spectral properties shown on Figure 33(a) satisfy the 3GPP specified values of ACLR at the 5 $\mathrm{MHz}$ and $10 \mathrm{MHz}$ offsets.

The variation of the measured received optical power and the W-CDMA signal ACLR characteristics is shown in Figure 33(b). Two cases are considered i.e. first case is back-toback (B-to-B) measurement using the RoF modules, signal generator and analyzer and an optical attenuator for incrementing the attenuation to represent channel losses and in the offsets. The B-to-B actual transmission over the RoFSO system measurements shows almost similar characteristics and the minimum optical received power to satisfy the prescribed 3GPP value at $5 \mathrm{MHz}$ at $10 \mathrm{MHz}$ offsets is about $-15 \mathrm{dBm}$. Using a post EDFA the required received optical power can be even as low as $-25 \mathrm{dBm}$ and $-20 \mathrm{dBm}$ and still satisfy the 3GPP specification for W-CDMA signal transmission at $5 \mathrm{MHz}$ and $10 \mathrm{MHz}$ offsets respectively. 


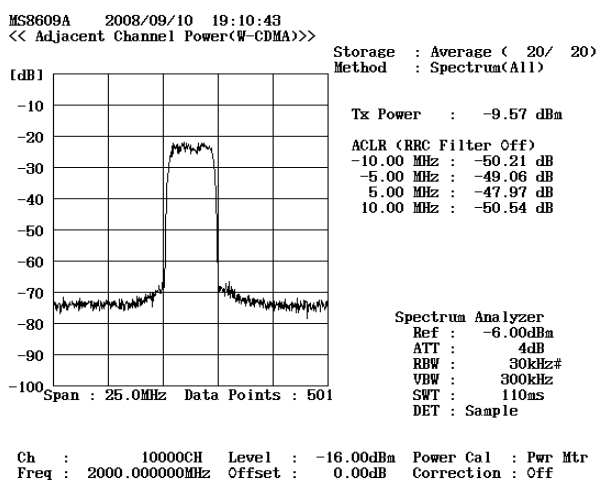

(a)

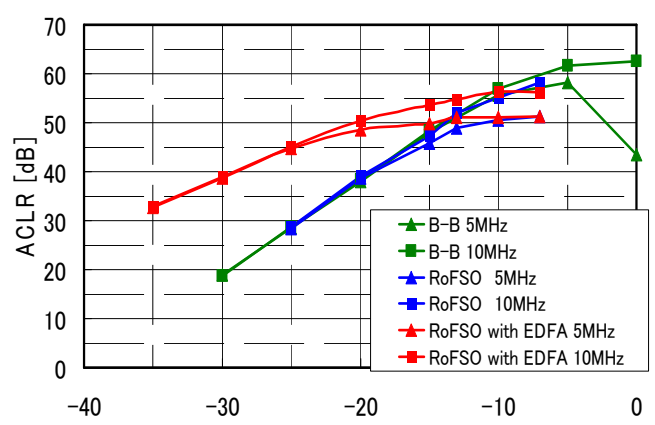

RoFSO Rx. Optical Power $[\mathrm{dBm}]$

(b)

Figure 33. (a) Received W-CDMA signal ACLR spectrum and (b) variations of ACLR and optical received power

\subsubsection{Wireless LAN}

In another example the RoFSO system is evaluated by transmitting a WLAN IEEE802.11 based signal. In this experiment, an IEEE802.11g compliant waveform is generated by a vector signal generator at $-24 \mathrm{dBm}$ which is applied to the RoF module in the RF interface unit. After transmission through the RoFSO link a spectrum analyzer is utilized to measure and analyze the quality of the received WLAN signal. A pass/fail judgment of the spectrum mask as defined in the IEEE specification $802.11 \mathrm{a} / \mathrm{b} / \mathrm{g}$ is used. As a test signal, IEEE802.11g waveform at $2.4 \mathrm{GHz}$ with $54 \mathrm{Mbps}$ 64QAM is used. Figure 34(a) depicts a WLAN signal with spectrum mask in this case. A constellation graph of the WLAN signal modulation analysis is shown in Figure 34(b).

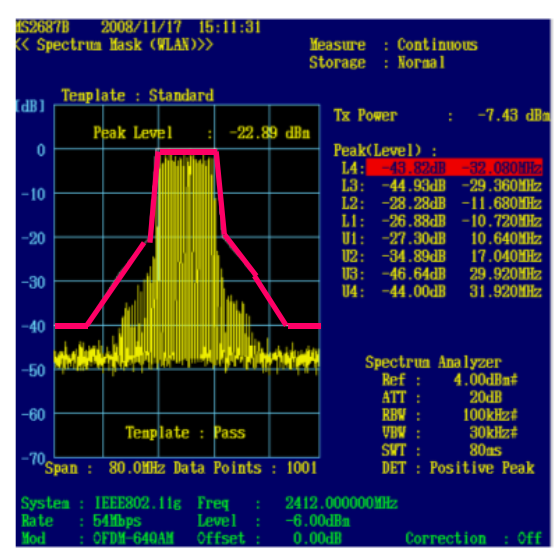

(a)

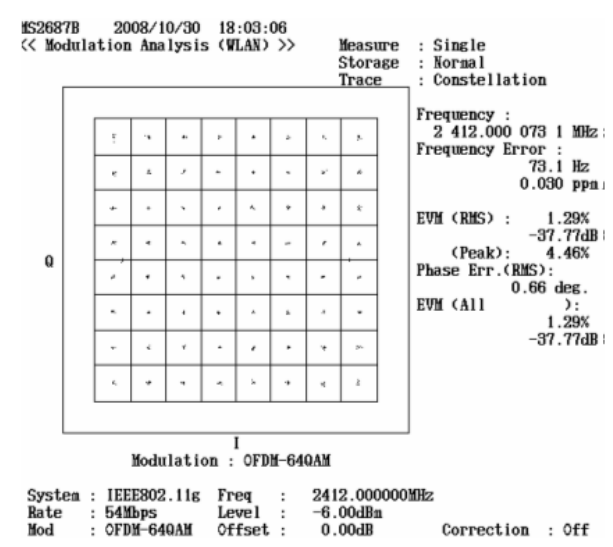

(b)

Figure 34. WLAN (a) spectrum mask and (b) modulation analysis constellation 
The recorded RMS of Error Vector Magnitude (EVM) value is within the acceptable tolerance for WLAN signal transition. The result of continuous measurement of the spectral mask test collected over two days is shown in Figure 35. The mask pattern Pass/Fall judgment is measured per second and recorded. This result is accumulated for every 1 minute, and the passed number is plotted in the figure. Moreover, in order to evaluate the variation with respect to the condition of the propagation path, the metrological data such as visibility and rain rate data which is simultaneously collected is plotted.

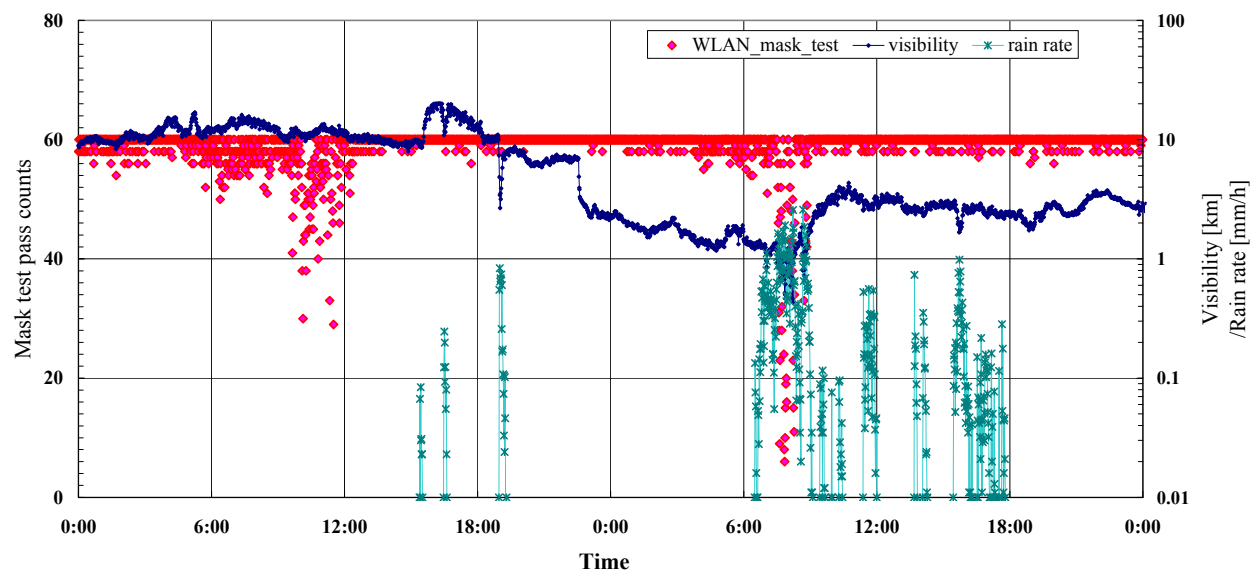

Figure 35. Mask test pass counts per 1 min. vs. weather condition

The measurement data depicted in the figure represent a fine weather condition before noon on the first day which later turned cloudy. On the second day it rained. The drop in visibility because of rain is significant around 8:00am on the second day. Because of the rain there is an increase in attenuation in the propagation path, so the received optical signal power falls therefore increasing the rate of the spectrum mask test failure. It can be observed in the figure that on the first day, there is an increase of spectrum mask failure rate around noon, this is due to the effect of atmospheric turbulence.

\subsubsection{ISDB-T (Digital TV)}

Digital terrestrial television broadcasting, referred to as Integrated Service Digital Broadcasting - Terrestrial (ISDB-T) in Japan, is designed to provide reliable high-quality video, sound and data broadcasting not only for fixed receivers but also for mobile receivers. The system is designed to provide flexibility, expandability and interoperability for multimedia broadcast. The ISDB-T system uses the UHF band at frequencies between $470 \mathrm{MHz}$ and $770 \mathrm{MHz}$, giving a total bandwidth of $300 \mathrm{MHz}$. The bandwidth is divided into 50 channels named from 13 to 62 . Each channel is further divided into 13 OFDM segments which includes a single segment, (A-Layer or 1seg), for mobile receivers (LDTV, 
audio and data) and the remainder can be allocated as one 12-segment for high definition television (HDTV) programs [DiBEG]. In this setup, channel 32 is used for ISDB-T signal transmission. A vector signal generator (Anritsu MG3700A) is used to output simple BER data and video waveforms for ISDB-T transmission evaluation. In this example, two signals are set simultaneously with the following waveform patterns (a) ISDBT_16QAM_1_2 (A-Layer: 1seg, 16QAM and B-Layer: 12seg, 64QAM) and (b) ISDBT_2layer_Movie, both at $-20 \mathrm{dBm}$ with a $6 \mathrm{MHz}$ frequency offset. The combined signal at $-17 \mathrm{dBm}$ is fed into the RoF module. The optical modulation index (OMI) for each channel (at $-20 \mathrm{dBm}$ input) is $10 \%$. The signal is subsequently transmitted over the RoFSO link. At the receiving site a digital broadcasting signal analyzer (Anritsu MS8901A) is used to measure the quality of the received ISDB-T signal. A received signal spectrum showing the two transmitted ISDB-T signals is depicted in Figure 36(a). A modulation error ratio (MER) quality metric parameter used to evaluate the modulation signal quality of the digital terrestrial television broadcasting signal directly and quantitatively is measured and analyzed. An example of modulation analysis constellation for the digital terrestrial broadcasting signal made of A-Layer 16QAM and B-Layer 64QAM is shown in Figure 36(b) and 36 (c) respectively which were captured when the recorded average received optical power was $-5.92 \mathrm{dBm}$ and $-5.88 \mathrm{dBm}$ respectively (i.e. the monitor output measured power adjusted for the respective RF signal). The constellation is very useful for analyzing the condition of the received signal by monitoring the modulation symbol movement. In Figure 36(b) and 36(c) the received signals exhibit little signal distortion (in terms of amplitude or frequency fluctuations) and the signal deterioration is minimal thus confirming the suitability of the RoFSO system for ISDB-T signal transmission conforming to the specified standard [ARIB]. In this example the measurement was made in the evening after 20:00 hrs considered to be weak atmospheric turbulence conditions. The ISDB-T signal transmission using the RoFSO system is also evaluated using a BER quality metric parameter. Figure 37 shows the BER measurement and the mean received optical power characteristics for one-segment (1seg) and 12-segment transmission collected over a 24 hour period on 11 December 2008. Increased bit errors are observed at around midday because of increase in the atmospheric turbulence which affects the received optical power. The variation of the measured mean received optical power can be correlated with the BER in this case. For 1seg transmission (A-Layer) the BER characteristics shows satisfactory performance with most values being below the error correction limit $\left(2 \times 10^{-4}\right)$ demonstrating the suitability of the RoFSO system for ISDB-T 1seg transmission. Unfortunately, occasionally the automatic gain control (AGC) is inadequate in the case for 12-segment HDTV (B-Layer) transmission. As an example the ISDB-T transmitted video captured screen shots for 1seg and 12-segment are shown in Figure 38(a) and 38(b) respectively. The 1 seg video quality was consistently clear and continuous without any stoppages. However, the 12-segment video is clear and continuous only in the absence of atmospheric turbulence or other effects which contribute to the deterioration of the transmitted RoFSO signal quality. 


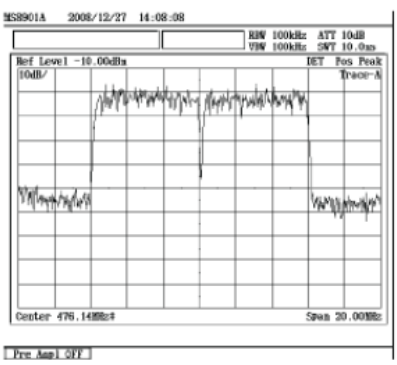

(a)

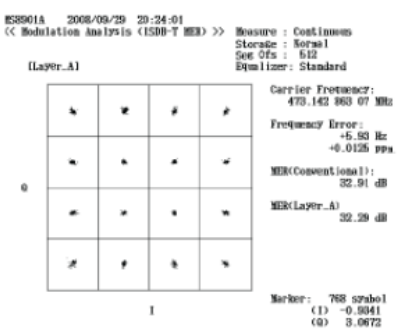

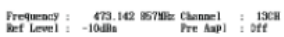

(b)

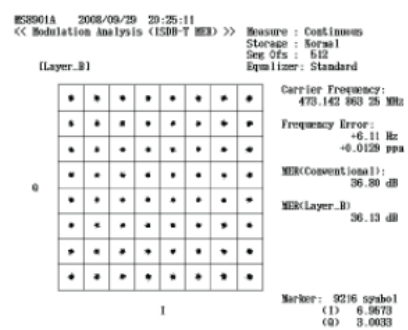

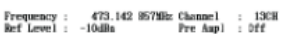

(c)

Figure 36. ISDB-T signal transmission experiment (a) received ISDB-T signal spectrum and modulation analysis constellations for (b) A-Layer 1 Seg (16QAM) and (c) B-Layer (64QAM)

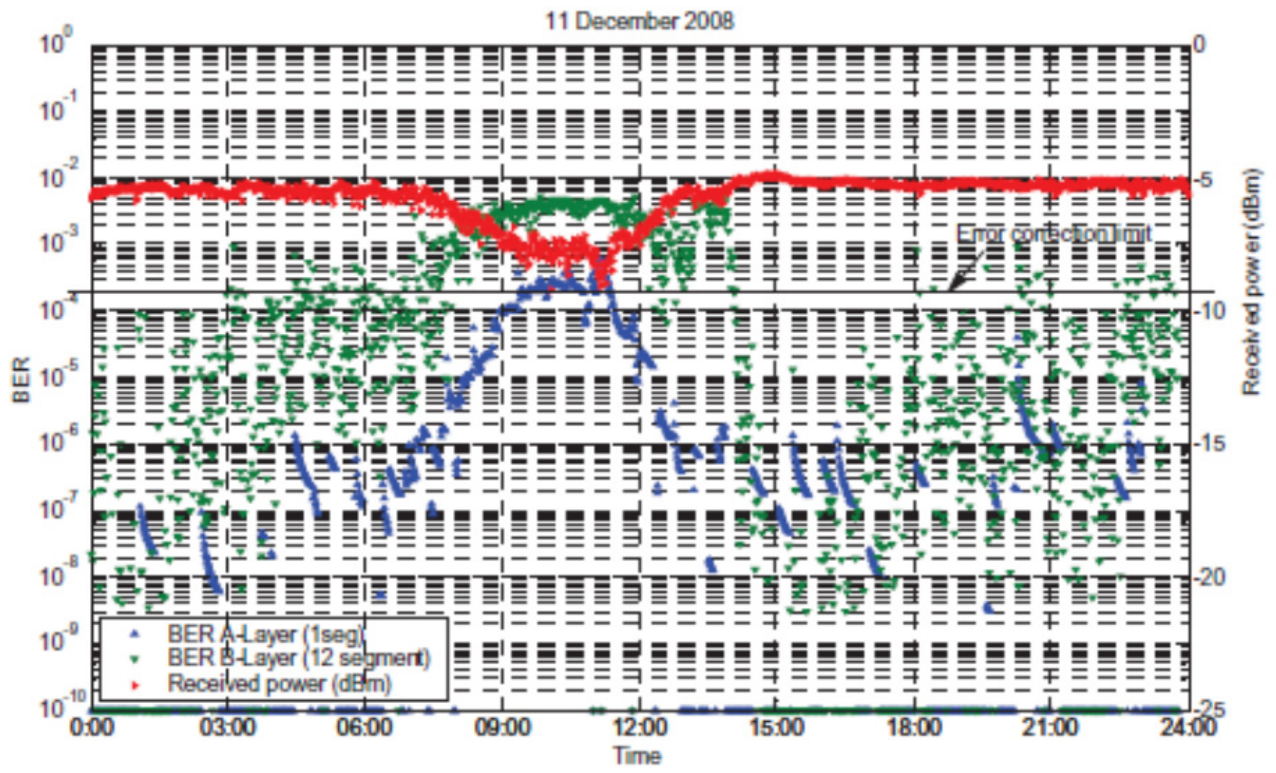

Figure 37. Digital terrestrial television broadcasting BER and received optical power characteristics 


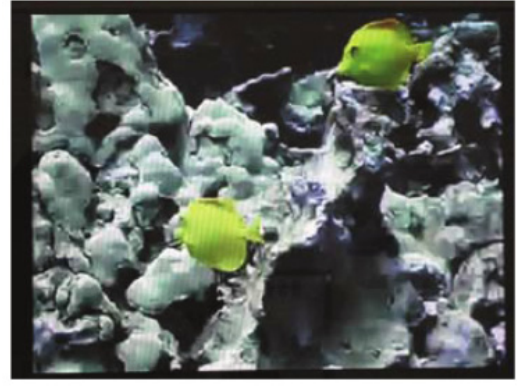

(a)

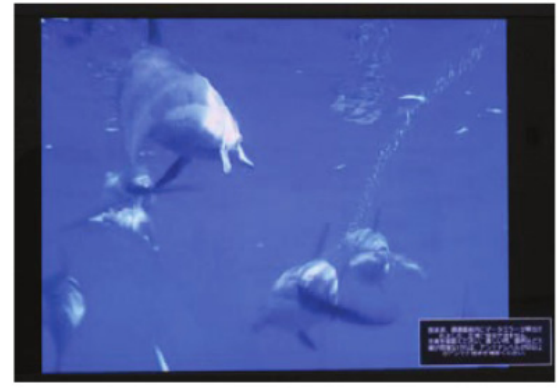

(b)

Figure 38. ISDB-T captured video screen shots (a) A-Layer 1seg and (b) B-Layer 12-segment

\subsection{Conclusions of the RoFSO system}

Simultaneous transmission of different kinds of wireless services using a newly developed RoFSO system has been presented. As the result, we have been presented that an all-optical connection FSO system could treat not only digital signal transmission but RF signal on a par with an optical fiber. And it is shown that the new generation optical wireless optical communication technology has a possibility of uniting radio environment and cable environment also with a service level, and the thing expectable as a solution of a heterogeneous network is shown.

\section{Conclusions}

We have described the concept and technology for the next-generation optical wireless communication systems. We explained the optics design method and the design results of the optical antennas considering laser propagation phenomena such as the scintillation and arrival beam angle fluctuation which occurs by atmospheric turbulence. We also mentioned the fine tracking mechanism using the FPM for the optical antennas. The FSO system incorporating high speed and highly precise tracking mechanism in which the influence of angle of arrival change is compensated succeed in maintaining free-space to SMF stably. A free-space optical communication system using specially designed compact antenna for easy, cost effective means of constructing a robust and reliable high-speed link for next generation optical wireless communication system was developed and investigated. The actual proof experiment using the developed NG-FSO system shows that this system enabled offer of a link equivalent to the fiber independent of the bit rate or a transmission protocol. Furthermore, we tried to unite this system with a RoF system and also enabled offer of various wireless services further. RoFSO system which expands RoF technology to a free-space using all-optical connection technology also shows the possibility of RF signal transmission through the field experiment. As these results, a next-generation optical wireless communication system using all-optical connection technology shows a possibility 
of becoming an effective solution of extension of a next-generation optical fiber system and heterogeneous wireless service.

\section{Author details}

Koichi Takahashi

Future Creation Laboratory, Olympus Corporation, Japan

\section{Acknowledgement}

I am indebted to Mr. Y. Miyazaki, Mr. T. Sakamoto, Ms. J. Aruga, Mr. T. Suzuki and Dr. Y. Arimoto for the help and support during NG-FSO system development and experiment and to Dr. M. Matumoto, Dr. K. Wakamori, Dr. K. Kazaura, Mr. T. Suzuki, Dr. S. Komaki, Dr. K. Tukamoto, and Dr. T. Higashino for NG-FSO system and RoFSO system experiment and evaluation.

This work is supported by a grant from the National Institute of Information and Communications Technology (NICT) of Japan.

\section{References}

[Cochennec 2002] J-Y Cochennec, “Activities on Next-Generation Networks Under Global Information Infrastructure in ITUT," IEEE Communications Magazine, vol. 40, pp. 98101, July 2002.

[ITU-T 2001] ITU-T Rec. Y.2001, “General overview of NGN,” Dec. 2004.

[ITU-T 2011] ITU-T Rec. Y.2011, “General principles and general reference model for Next Generation Networks,"Oct. 2004.

[Lee 2007] S-Q. Lee, et al., "The wireless broadband (wibro) system for broadband wireless internet services," IEEE Communications Magazine, vol. 44, pp. 106-112, July 2007.

[Ben 2006] S. J. Ben Yoo, "Optical Packet and Burst Switching Technologies for the Future Photonic Internet, " J. Lightwave Technol. 24, 4468-4492 (2006)

[Zhang 2005] Y. Zhang, O. W. Yang and Y. Zhai, "The optimization issues in an agile allphotonic backbone network, " Proc. SPIE 5626, 1233 (2005)

[Kitayama 2005] K. Kitayama, T. Miki, et al., "Photonic Network R\&D Activities in JapanCurrent Activities and Future Perspectives," J. Lightwave Technol. 23, 3404- (2005)

[Kim 1999] I. I. Kim, M. Mitchel, and E. Korevaar, "Measurement of Scintillation for Freespace Laser Communication at 785nm and 1550nm," in Optical Wireless Communications II, vol. 3850, (Boston, Massachusetts), pp. 49-62, SPIE, Sept. 1999.

[Bouchet 1999] O. Bouchet, H. Sizun, et al., "Free-Space Optics Propagation and Communication." ISTE, 2006.assachusetts), pp. 49-62, SPIE, Sept. 1999.

[Kaliski 1999] R. W. Kaliski, S. M. Genco, et al., "Laser communication intersatellite links realized with commercial off-the-shelf technology," in Free-Space Laser 
Communication Technologies XI (G. S. Mecherle, ed.), vol. 3615, pp. 170-178, SPIE, Apr. 1999.

[Borocom 2005] D.M.Borocom, C.C.Chan and B.Edwards, "Overview of the Mars laser communications demonstration Project," IEEE LEOS Newslett., 19 No.5(2005) 8-11

[Koyama 2004] Y. Koyama, E. Morikawa, et al., "Optical terminal for NeLS in-orbit demonstration," in Free-Space Laser Communication Technologies XVI (G.S.Mecherle, C. Y. Young, and J. S. Stryjewski, eds.), vol. 5338, (San Jose, CA, USA), pp. 29-36, SPIE, June 2004.

[Infrared] Infrared Communication Systems Association. http://www.icsa.org.jp.

[Free-sSpace] Free-Space Optical Alliance. http://www.wcai.com/fsoalliance.

[Takahashi 2008] K. Takahashi and Y. Arimoto, “Compact optical antennas using free-form surface optics for ultrahigh-speed laser communication systems," SPIE Optical Engineering 47(1), pp. 016002- 1-9, 2008.

[Matsumoto 2008] M. Matsumoto, K. Kazaura, P. Dat, et al., “An alternative access technology for next generation networks based on full-optical wireless communication links," in Kaleidoscope-Innovations in NGN: Future Network and Services (K-INGN 2008), pp. 221 -228, May 2008. Proc. of SPIE Vol. 7199 719907-11

[Kazaura 2007] K. Kazaura, K. Omae, T. Suzuki, et al., "Performance Evaluation of Next Generation Free-Space Optical Communication System," IEICE Transactions on Electronics E90-C, pp. 381-388, Feb. 2007.

[Khaleghi 1996] F. Khaleghi, J. Li, M. Kavehrad, and H. Kim, "Increasing Repeater Span in High-speed Bidirectional WDM Transmission Systems Using a New Bidirectional EDFA Configuration," IEEE Photon. Technol. Lett., vol. 8, no. 9, 1996, pp. 1252-1254.

[Luo 1998] G. Luo, J.L. Zyskind, J.A. Nagel, and Mohamed A. Ali, "Experimental and Theoretical Analysis of Relaxation-Oscillations and Spectral Hole Burning Effects in AllOptical Gain-Clamped EDFA's for WDM Networks", IEEE J. Lightwave Technology, vol. 16, no. 4, 1998, pp. 527-529.

[Al-Raweshidy 2002] H. Al-Raweshidy and S. Komaki, eds., "Radio Over Fiber Technologies for Mobile Communications Networks. "Artech House Publishers, 1 ed., 2002.

[Hai 2006] L. H. Hai, et al., "Performance improvement of radio-on-fiber ubiquitous antenna system using sub-carrier resource management, " Proc. SPIE 6390, 63900B (2006)

[Komaki 2003] S. Komaki, "Microwave Technologies for Software Radio Networks", Proceedings of APMC2003, Vol.3, No.FC7-1, pp.1780-1785, (November 2003).

[Tsukamoto 2005] K. Tsukamoto, "Software Definable Radio Networks for the Ubiquitous Networks", XXXIIIth GA of URSI, vol.1,p.205, (October 2005).

[Tatarskii 1997] V.I.Tatarskii, "The Effects of the Turbulent Atmosphere on Wave Propagation, U.S.Dept. of Commerce", National Technical Information Service, Springfield, VA, 1971.

[Dayton 1992] D.Dayton, B.Pierson, B.Spielbusch, J.Gonglewski, “Atomospheric structure function measurements with a Shack-Hartmann wave-front sensor," Opt. lett. 17,1737,1992. 
[Hufnagel 1974] R.E.Hufnagel, Proc. Top. Mtg. on Opt., “Propagation through turbulence," Boulder, CO 1974

[Fried 1967] D. L. Fried and J. B. Seidman, "Laser beam scintillations in the atmosphere," J. Opt. Soc. Am. 57, 181-185 (1967)

[Andrew 1998] L. C. Andrews and R. L. Phillips, "Laser Beam Propagation through Random Media," SPIE Optical Engineering Press, Bellingham, WA, (1998).

[Andrew 1995] L. C. Andrews, R. L. Phillips and P. T. Yu: "Optical scintillations and fade statistics for a satellite-communication system," Applied Optics, Vol. 34, No. 33 (1995).

[Arimoto 2003] Y.Arimoto, M.Nagatsuka, et al., "Laser Communication Experiment Using Stratospheric Platform System," Technical Report of IEICE SANE2003-82 (2003-11).

[Katsuo 2005] F. Katsuo, H. Kikuchi, et al., “Wireless System Demonstration Trial Using a Low-Altitude Station-Keeping Test Airship Optical Communications Experiment,'Technical Report of IEICE SANE2004-81(2005-01)

[Takahashi 2006] K. Takahashi and Y. Arimoto, "Development of optical antennas utilizing free form surface optics for the high speed laser communication systems," Free-Space Laser Communication Technologies XVIII, vol.6105, pp.40-49, SPIE, Jan. 2006.

[Takahashi 2011] K. Takahashi, "Development of Ultrawide-Angle Compact Camera Using Free-Form Optics," OPTICAL REVIEW Vol.18,No.1 (2011)55-59

[Kazaura 2006] K. Kazaura, K. Omae, T Suzuki, et al., "Mitigation of atmospheric effects on terrestrial FSO communication systems by using high-speed beam tracking antenna", Free-Space Laser Communication Technologies XVIII, 24 25 Jan 2006, San Jose, California, USA

[Arimoto 2007] Y. Arimoto, "Multi-gigabit Free-space Optical Communication System with Bidirectional Beacon Tracking," IEEJ Trans. FM, vol. 127, no. 7, pp. 385-390, 2007.

[Kazaura 2009] K. Kazaura, P. Dat, A. Bekkali, et al., “Experimental evaluation of a Radioon-FSO communication system for multiple RF signal transmission", Paper 7199-6, Photonics West 2009, SPIE, San Jose, 2009

[Kim 2009] K-H. Kim, T. Higasino, et al., "Performance evaluation of WLAN signal transmission by using a new statistical model of RoFSO Channel, " 1st International Conference on Space Optical Systems and Applications (ICSOS 2009), ICSOS2009-33, Miraikan, Tokyo, Japan, 4-6 Feb. 2009.

[Tsukamoto 2008] K. Tsukamoto, H. Onodera, et al., "Project of Radio on Free Space Optic System Development for Heterogeneous Wireless Services," IPGC 2008 IEEE Photonics Global@Singapore, pp: 1-4 8-11 Dec. 2008

[Takahashi 2008] K. Takahashi, T. Higashino, et al., “Design and evaluation of optical antenna module suitable for radio-on free-space optics link system for ubiquitous wireless", Proceedings of SPIE for Photonic West, vol. 6877, pp. 68770H_1-11, San Jose, California USA, Jan 2008.

[Wakamori 2008] K. Wakamori, K. Kazaura, et al., "An Experimental Study of Next Generation FSO Systems, " IEICE TRANS Commu, Vol. J91-C, No. 1, pp. 28-37, Jan. 2008 
[3GPP 2002] 3GPP TS 25.141, 3rd Generation Partnership Project. http://www.3gpp.org/, 2002.

[DiBEG] DiBEG. http://www.dibeg.org/.

[ARIB] ARIB STD-B31. http://www.arib.or.jp/english/html/overview/ov/std_b31.html. 